\title{
Simulation of the Radiation Reaction Orbits of a Classical Relativistic Charged Particle with Generalized Off-Shell Lorentz Force
}

\author{
Aviad Roitgrund ${ }^{1}$ and Lawrence Horwitz ${ }^{1,2,3}$ \\ ${ }^{1}$ School of Physics, Tel Aviv University, Ramat Aviv 69978, Israel \\ ${ }^{2}$ Department of Physics, Bar Ilan University, Ramat Gan 52900, Israel \\ ${ }^{3}$ Physcis Department, Ariel University Center of Sameria, Ariel 40700, Israel \\ Correspondence should be addressed to Lawrence Horwitz, larry@post.tau.ac.il \\ Received 2 June 2010; Accepted 4 October 2010 \\ Academic Editor: Marko Robnik
}

Copyright (c) 2010 A. Roitgrund and L. Horwitz. This is an open access article distributed under the Creative Commons Attribution License, which permits unrestricted use, distribution, and reproduction in any medium, provided the original work is properly cited.

\begin{abstract}
We review the formulation of the problem of electromagnetic self-interaction of a relativistic charged particle in the framework of the manifestly covariant classical mechanics of Stueckeleberg, Horwitz, and Piron. The gauge fields of this theory, in general, cause the mass of the particle to change. We study the four dynamical off-mass-shell orbit equations which result from the expansion of Green's function in the Lorentz force equation for the self-interaction. It appears that there is an attractor in this system which stabilizes the motion of the relativistic charged electron. The attractor may acquire fractal characteristics in the presence of an external field and thus become a strange attractor.
\end{abstract}

\section{Introduction}

This work is concerned with studying the problem of the classical relativistic charged particle. The existence of radiation due to the accelerated motion of the particle raises the question of how this radiation, acting back on the particle, affects its motion. Rohrlich has described the historical development of this problem, where the first steps were taken by Abraham in 1905, culminating in the work of Dirac, who derived the equation for the ideal point electron in the form

$$
m \frac{d^{2} x^{\mu}}{d s^{2}}=\frac{e}{c} F_{v}^{\mu} \frac{d x^{v}}{d s}+\Gamma^{\mu}
$$


where $m$ is the electron mass, including electromagnetic correction, $s$ is the proper time along the trajectory $x^{\mu}(s)$ in spacetime, $F_{v}^{\mu}$ is the covariant form of the electromagnetic force tensor, $e$ is the electron charge, and

$$
\Gamma^{\mu}=\frac{2}{3} \frac{e^{2}}{c^{3}}\left(\frac{d^{3} x^{\mu}}{d s^{3}}-\frac{d^{2} x^{v}}{d s^{2}} \frac{d^{2} x_{v}}{d s^{2}} \frac{d x^{\mu}}{d s}\right)
$$

Here, the indices $\mu, v$, running over $0,1,2,3$ label the spacetime variables that represent the action of the Lorentz group; the index raising and lowering Lorentz invariant tensor $\eta_{\mu \nu}$ is of the form $\operatorname{diag}(-1,+1,+1,+1)$. The expression for $\Gamma^{\mu}$ was originally found by Abraham in 1905 [1], shortly after the discovery of special relativity and is known as the Abraham four-vector of radiation reaction. Dirac's derivation [2] was based on a direct application of Green's functions for the Maxwell fields, obtaining the form (1.1), the Abraham-LorentzDirac equation. In this calculation, Dirac used the difference between retarded and advanced Green's functions so as to eliminate the singularity carried by each. Sokolov and Ternov [3], for example, give a derivation using the retarded Green's function alone and show how the singular term can be absorbed into the mass $m$.

The formula (1.1) contains the so-called singular perturbation problem. There is a small coefficient multiplying a derivative of higher order than that of the unperturbed problem; since the highest-order derivative is the most important in the equation, one sees that dividing by $e^{2}$, any small deviation in the lower order terms results in a large effect on the orbit, and there are unstable solutions, often called "runaway solutions". There is extensive literature (see [4-6] and the references therein) on the methods of treating this instability.

The existence of these "runaway solutions", for which the electron undergoes an exponential acceleration with no external force beyond a short initial perturbation of the free motion, is a difficulty for the point electron picture in the framework of the Maxwell theory with the covariant Lorentz force. Rohrlich [7-9] has discussed the idea that the point electron idealization may not be really physical, based on arguments from classical and quantum theory and emphasized that for the corresponding classical problem, a finite size can eliminate this instability. Of course, this argument is valid, but it leaves open the question of the consistency of the Maxwell-Lorentz theory which admits the concept of point charges.

Stueckelberg, in 1941 [10-14], proposed a manifestly covariant form of classical and quantum mechanics in which space and time become dynamical observables. They are therefore represented in quantum theory by operators on a Hilbert space on square integrable functions in space and time. The dynamical development of the state is controlled by an invariant parameter $\tau$, which one might call the world time, coinciding with the time on the (on mass shell) freely falling clocks of general relativity.

Stueckelberg postulated the existence of an invariant "Hamiltonian" $K$, for the classical theory, which would generate Hamilton equations for the canonical variables $x^{\mu}$ and $p^{\mu}$ of the form (we take units for which $c=1$ unless otherwise specified)

$$
\begin{gathered}
\dot{x}^{\mu}=\frac{\partial K}{\partial p_{\mu}}, \\
\dot{p}^{\mu}=-\frac{\partial K}{\partial x_{\mu}}
\end{gathered}
$$


where the dot indicates differentiation with respect to $\tau$. Taking, for example, the model

$$
K_{0}=\frac{p^{\mu} p_{\mu}}{2 M}
$$

we see that the Hamilton equations imply that

$$
\dot{x}^{\mu}=\frac{p^{\mu}}{M}
$$

It then follows that

$$
\frac{d \vec{x}}{d t}=\frac{\vec{p}}{E}
$$

where $p^{0} \equiv E$, and we set the velocity of light $c=1$; this is the correct definition for the velocity of a free relativistic particle. Moreover, it follows that

$$
\dot{x}^{\mu} \dot{x}_{\mu}=\frac{d x^{\mu} d x_{\mu}}{d \tau^{2}}=\frac{p^{\mu} p_{\mu}}{M^{2}} .
$$

With our choice of metric, $d x^{\mu} d x_{\mu}=-d s^{2}$ and $p^{\mu} p_{\mu}=-m^{2}$, where $m$ is the classical experimentally measured mass of the particle (at a given instant of $\tau$ ). We see from this that

$$
\frac{d s^{2}}{d \tau^{2}}=\frac{m^{2}}{M^{2}}
$$

and hence the proper time is not identical to the evolution parameter $\tau$. In the case where $m^{2}=M^{2}$, it follows that $d s=d \tau$, and we say that the particle is "on shell".

For example, in the case of an external potential $V(x)$, where we write $x \equiv x^{\mu}$, the Hamiltonian becomes

$$
K=\frac{p^{\mu} p_{\mu}}{2 M}+V(x)
$$

so that, since $K$ is a constant of the motion, $\mathrm{m}^{2}$ varies from point to point with the variations of $V(x)$. It is important to recognize from this discussion that the observable particle mass depends on the state of the system (in the quantum theory, the expectation value of the operator $p^{\mu} p_{\mu}$ provides the expected value of the mass squared).

Since, in nature, particles appear with fairly sharp mass values (not necessarily with zero spread), we may assume the existence of some mechanism which will drive the particle's mass back to its original mass-shell value (after the source responsible for the mass change ceases to act) so that the particle's mass shell is defined. We will not take such a mechanism into account explicitly here in developing the dynamical equations. We will assume that if this mechanism is working, it is a relatively smooth function (e.g., a minimum in free energy which is broad enough for our off-shell driving force to work fairly freely) (A relativistic 
Lee model has been worked out which describes a physical mass shell as a resonance, and therefore a stability point on the spectrum [15], but at this point it is not clear to us how this mechanism works in general.).

The Stueckelberg formulation implies the existence of a fifth "electromagnetic" potential, through the requirement of gauge invariance, and there is a generalized Lorentz force which contains a term that drives the particle off shell, whereas the terms corresponding to the electric and magnetic parts of the usual Maxwell fields do not (for the nonrelativistic case, the electric field may change the energy of a charged particle, but not the magnetic field; the electromagnetic field tensor in our case is analogous to the magnetic field, and the new field strengths, derived from the $\tau$ dependence of the fields and the additional gauge field, are analogous to the electric field, as we will see).

In Section 2, we give the structure of the field equations and show that the standard Maxwell theory is properly contained in this more general framework.

In Section 3, we apply Green's functions to the current source provided by the relativistic particle. We obtain the equations of motion for a relativistic particle which is, in general, off-shell. As in Dirac's result, these equations are of third order in the evolution parameter and therefore are highly unstable. Our results exhibit what appears to be an attractor in this system.

We introduce the quantity

$$
\varepsilon=1+\dot{x}^{\mu} \dot{x}_{\mu}
$$

which measures the deviation of the motion of the charged particle from its "mass shell", where we used the metric $(-,+,+,+)$ for the four-vector product.

In Section 4, we derive an autonomous mass deviation equation (with coefficients independent of $\tau$ or $x^{\mu}$ and its derivatives) for the development of $\varepsilon$ in the specific case of a particle under the influence of self-interaction in the absence of external field.

When the formal derivation of the autonomous equation is complete, a numerical analysis follows in Section 5 and demonstrates the compatibility between the (four component) dynamical equation of motion and the autonomous equation.

In Sections 6 and 7, we analyze the motion of the (charged) particle under the influence of self-interaction in the absence of external fields. We show that when initial conditions are within a basin of attraction, the trajectory of a particle obeying the equations of motion developed in Sections 3 and 4 forms an attractor.

We further show that the attractor has a degree of stochasticity which is dictated by the cutoff frequency one chooses. A closer to zero (and thus more physical) cutoff frequency causes fluctuations to appear in the attractor, while a higher cutoff frequency results in a nonfluctuating attractor. There is an opposite relation between stochasticity and stability. Thus, a stochastic attractor has shorter life span. However, even an attractor with a relatively high cutoff frequency (and hence a longer life span) eventually ceases to exist as the orbit runs away to infinity.

Our analysis shows that the effective mass of the electron grows as it propagates towards the vicinity of $\varepsilon=1$ (the light cone). This growth can be seen either through the growing amplitude of the effective mass in the case of a relatively low cutoff frequency or through a nonfluctuating growth of the effective mass (absolute) value in the case of a relatively high cutoff frequency.

We demonstrate that the growing effective mass stabilizes the effect of the generalized Lorentz force. The demonstration consists of a correlation between the growing effective 
mass, the growing trajectory stability (measured through calculating the Lyapunov exponent), and the decay of acceleration and velocity.

The chaotic nature of the electron's trajectory is also checked, using two methods: the (mentioned above) Lyapunov exponent calculation and a time series analysis. As will be shown, the results do not indicate a chaotic nature of the orbit, even in fluctuating cases.

However, the nature of the attractor changes in the presence of an external field, as shown in Section 8. In that section we show some results with remarkable stochasticity. This is reflected in the phase space graphs of the dynamical equation of motion, which show fractal behavior. Thus, the simple attractor of the pure self-interaction becomes a strange attractor (due to the fractal nature) in the presence of an external field. Experiments with high-frequency detectors may pick up such stochastic-type signals.

\section{Equations of Motion}

The Stueckelberg-Schrödinger equation which governs the evolution of a quantum state over the manifold of spacetime was postulated by Stueckelberg [10-14] to be, for the free particle,

$$
i \frac{\partial \psi_{\tau}}{\partial \tau}=\frac{p^{\mu} p_{\mu}}{2 M} \psi_{\tau}
$$

where $i p_{\mu}$ is represented by $\partial / \partial x^{\mu} \equiv \partial_{\mu}$ (we take $\hbar=1$ ).

Taking into account full $U(1)$ gauge invariance, corresponding to the requirement that the theory maintain its form under the replacement of $\psi$ by $e^{i e_{0} \Lambda} \psi$, the StueckelbergSchrödinger equation (including a compensation field for the $\tau$-derivative of $\Lambda$ ) [16-18] is

$$
\left(i \frac{\partial}{\partial \tau}+e_{0} a_{5}(x, \tau)\right) \psi_{\tau}(x)=\frac{\left(p^{\mu}-e_{0} a^{\mu}(x, \tau)\right)\left(p_{\mu}-e_{0} a_{\mu}(x, \tau)\right)}{2 M} \psi_{\tau}(x)
$$

where the gauge fields may depend on $\tau$ and $e_{0}$ is a coupling constant which we will see having the dimension $l^{-1}$. The corresponding classical Hamiltonian then has the form

$$
K=\frac{\left(p^{\mu}-e_{0} a^{\mu}(x, \tau)\right)\left(p_{\mu}-e_{0} a_{\mu}(x, \tau)\right)}{2 M}-e_{0} a_{5}(x, \tau)
$$

in place of (2.1). Stuckelberg did not take into account this full gauge invariance requirement, working in the analog of what is known in the nonrelativistic case as the Hamilton gauge (where the gauge function $\Lambda$ is restricted to be independent of time) and therefore had some difficulty in accounting for pair creation and annihilation in an electromagnetic field. The equations of motion for the field variables are given (for both the classical and quantum theories) by

$$
\lambda \partial_{\alpha} f^{\beta \alpha}(x, \tau)=e_{0} j^{\beta}(x, \tau)
$$


where $\alpha, \beta=0,1,2,3,5$, the last corresponding to the $\tau$ index and $\lambda$, of dimension $l^{-1}$, is a factor on the terms $f^{\alpha \beta} f_{\alpha \beta}$ in the Lagrangian associated with (2.2) (with, in addition, degrees of freedom of the fields) required by dimensionality. The field strengths are

$$
f^{\alpha \beta}=\partial^{\alpha} a^{\beta}-\partial^{\beta} a^{\alpha},
$$

and the current satisfies the conservation law [16-18]

$$
\partial_{\alpha} j^{\alpha}(x, \tau)=0
$$

Integrating over $\tau$ on $(-\infty, \infty)$ and assuming that $j^{5}(x, \tau)$ vanishes at $|\tau| \rightarrow \infty$, one finds that

$$
\partial_{\mu} J^{\mu}(x)=0,
$$

where [19]

$$
J^{\mu}(x)=\int_{-\infty}^{\infty} d \tau j^{\mu}(x, \tau)
$$

We identify this $J^{\mu}(x)$ with the Maxwell conserved current. In [20], for example, this expression occurs with

$$
j^{\mu}(x, \tau)=\dot{x}(\tau) \delta^{4}(x-x(\tau))
$$

and $\tau$ is identified with the proper time of particle (an identification which can be made for the motion of a free particle). The conservation of the integrated current then follows from the fact that

$$
\partial_{\mu} j^{\mu}=\dot{x}^{\mu}(\tau) \partial_{\mu} \delta^{4}(x-x(\tau))=-\frac{d}{d \tau} \delta^{4}(x-x(\tau))
$$

is a total derivative. We assume that the world line runs to infinity (at least in the time dimension) and therefore its integral vanishes at the end points [10-14, 20], in accordance with the discussion above.

As for the Maxwell case, one can write the current formally in five-dimensional form

$$
j^{\alpha}=\dot{x}^{\alpha} \delta^{4}(x(\tau)-x)
$$

For $\alpha=5$, the factor $\dot{x}^{5}$ is unity, and this component therefore represents the event density in spacetime.

Integrating the $\mu$ components of (2.4) over $\tau$ (assuming $f^{\mu 5}(x, \tau) \rightarrow 0$ pointwise for $\tau \rightarrow \pm \infty$ ), we obtain the Maxwell equation with the (dimensionless) Maxwell charge $e=$ $e_{0} / \lambda$ and the Maxwell fields given by

$$
A^{\mu}=\int_{-\infty}^{\infty} a^{\mu}(x, \tau) d \tau .
$$


A Hamiltonian in the form (2.3) without $\tau$ dependence of the fields, and without the $a_{5}$ terms, as written by Stuckelberg [10-14], can be recovered in the limit of the zero mode of the fields (with $a_{5}=0$ ) in a physical state for which this limit is a good approximation. This state occurs when the Fourier transform of the fields, defined by

$$
a^{\mu}(x, \tau)=\int_{-\infty}^{\infty} d s \widehat{a}^{\mu}(x, s) e^{-i s \tau}
$$

has support only in a small neighborhood $\Delta s$ of $s=0$. The vector potential then takes on the form $a^{\mu}(x, \tau) \approx \Delta s \widehat{a}^{\mu}(x, 0)=(\Delta s / 2 \pi) A^{\mu}(x)$, and we identify $e=(\Delta s / 2 \pi \lambda) e_{0}$. The zero mode therefore emerges when the inverse correlation length of the field $\Delta s$ is sufficiently small. We remark that in this limit, the fifth equation obtained from (2.4) decouples. The generalized Lorentz force obtained from this Hamiltonian, using the Hamilton equations, coincides with the usual Lorentz force, and, as we have seen, the generalized Maxwell equations reduce to the usual Maxwell equations. The theory therefore contains the usual Maxwell Lorentz theory in the zero mode; for this reason we have called this generalized theory the "pre-Maxwell" theory.

If such a pre-Maxwell theory really underlies the standard Maxwell theory, then there should be some physical mechanism which restricts most observations in the laboratory to be close to the zero mode. For example, in a metal there is a frequency, the plasma frequency, above which there is no transmission of electromagnetic waves. In this case, if the physical universe is imbedded in a medium which does not allow high "frequencies" to pass, the preMaxwell theory reduces to the Maxwell theory. Some study has been carried out, for a quite different purpose (of achieving a form of analog gravity), on the properties of the generalized fields in a medium with general dielectric tensor [21]. We will see in the present work that the high level of nonlinearity of this theory in interaction with matter may itself generate an effective reduction to Maxwell-Lorentz theory, with the high frequency chaotic behavior providing the regularization achieved by models of the type discussed by Rohrlich [7-9].

We remark that integration over $\tau$ does not bring the generalized Lorentz force into the form of the standard Lorentz force, since it is nonlinear, and a convolution remains. If the resulting convolution is trivial, that is, in the zero mode, the two theories then coincide. Hence, we expect to see dynamical effects in the generalized theory which are not present in the standard Maxwell-Lorentz theory.

Writing the Hamilton equations

$$
\dot{x}^{\mu}=\frac{d x^{\mu}}{d \tau}=\frac{\partial K}{\partial p_{\mu}}, \quad \dot{p}^{\mu}=\frac{d p^{\mu}}{d \tau}=-\frac{\partial K}{\partial x_{\mu}},
$$

for the Hamiltonian (2.3), we find that the generalized Lorentz force is

$$
M \ddot{x}^{\mu}=e_{0} f_{v}^{\mu} \dot{x}^{\nu}+f_{5}^{\mu}
$$

Multiplying this equation by $\dot{x}_{\mu}$, one obtains

$$
M \dot{x}_{\mu} \ddot{x}^{\mu}=e_{0} \dot{x}_{\mu} f_{5}^{\mu}
$$


This equation therefore does not necessarily lead to the simple relation between $d s$ and $d \tau$ discussed above in connection with (1.9). The $f_{5}^{\mu}$ term has the effect of moving the particle off-shell (as, in the nonrelativistic case, the energy is altered by the electric field). This can be seen by noting that

$$
\dot{x}^{\mu} \dot{x}_{\mu}=-\frac{m^{2}}{M^{2}}
$$

and the derivative is

$$
\dot{x}^{\mu} \ddot{x}_{\mu}=-\frac{1}{M^{2}} \frac{d}{d \tau} m^{2}=\frac{1}{M} e_{0} \dot{x}_{\mu} f_{5}^{\mu}
$$

or in another words,

$$
\frac{d}{d \tau} m^{2}=-M e_{0} \dot{x}_{\mu} f_{5}^{\mu}
$$

Let us now define

$$
\varepsilon=1+\dot{x}^{\mu} \dot{x}_{\mu}=1-\frac{d s^{2}}{d \tau^{2}}
$$

where $d s^{2}=d t^{2}-d \vec{x}^{2}$ is the square of the proper time. Since $\dot{x}^{\mu}=\left(p^{\mu}-e_{0} a^{\mu}\right) / M$ and $\left(p^{\mu}-\right.$ $\left.e_{0} a^{\mu}\right)\left(p_{\mu}-e_{0} a_{\mu}\right)=-m^{2}$ is interpreted as the gauge-invariant particle mass [16-18], then

$$
\varepsilon=1-\frac{m^{2}}{M^{2}}
$$

measures the deviation from "mass shell" (on mass shell, $d s^{2}=d \tau^{2}$ ).

\section{Derivation of the Differential Equations for the Spacetime Orbit with Off-Shell Corrections}

We now review the derivation of the radiation reaction formula in the Stueckelberg formalism (see also [19]). Calculating the self-interaction contribution, one must include the effects of the force acting upon the particle due to its own field $\left(f_{\text {self }}\right)$ in addition to the fields generated by other electromagnetic sources $\left(f_{\text {ext }}\right)$. Therefore, the generalized Lorentz force, using (2.14) takes the form:

$$
M \ddot{x}^{\mu}=e_{0} \dot{x}^{v} f_{\text {ext } v}^{\mu}+e_{0} \dot{x}^{v} f_{\text {self } v}^{\mu}+e_{0} f_{\text {ext } 5}^{\mu}+e_{0} f_{\text {self } 5}^{\mu}
$$


where the derivatives (dot) are with respect to the universal time $\tau$ and the fields are evaluated on the event's trajectory. Multiplying (3.1) by $\dot{x}_{\mu}$, we get the projected equation (2.16) in the form

$$
\frac{M}{2} \dot{\varepsilon}=e_{0} \dot{x}_{\mu} f_{\text {ext } 5}^{\mu}+e_{0} \dot{x}_{\mu} f_{\text {self } 5}^{\mu} .
$$

The field generated by the current is given by the pre-Maxwell equations (2.4), and choosing for it the generalized Lorentz gauge $\partial_{\alpha} a^{\alpha}=0$, we get

$$
\lambda \partial_{\alpha} \partial^{\alpha} a^{\beta}(x, \tau)=\lambda\left(\sigma \partial_{\tau}^{2}-\partial_{t}^{2}+\nabla^{2}\right) a^{\beta}=-e_{0} j^{\beta}(x, \tau),
$$

where $\sigma= \pm 1$ corresponds to the possible choices of metric for the $O(4,1)$ or $O(3,2)$ symmetry of the homogeneous field equations.

Green's functions for (3.3) can be constructed from the inverse Fourier transform

$$
G(x, \tau)=\frac{1}{(2 \pi)^{5}} \int d^{4} k d k \frac{e^{i\left(k^{\mu} x_{\mu}+\sigma k \tau\right)}}{k_{\mu} k^{\mu}+\sigma k^{2}} .
$$

Integrating this expression over all $\tau$ gives Green's function for the standard Maxwell field (treatment of the zeros in the denominator must be reconsidered after the $\tau$ integration). Assuming that the radiation reaction acts causally in $\tau$, we will use here the $\tau$-retarded Green's function. In this calculation of the Lorentz force, Dirac used the difference between advanced and retarded Green's functions in order to cancel the singularities that they contain. One can, alternatively, use the retarded Green's function and "renormalize" the mass in order to eliminate the singularity [3]. In this analysis, we follow the latter procedure.

The $\tau$-retarded Green's function [19] is given by multiplying the principal part of the integral (3.4) by $\theta(\tau)$. Carrying out the integrations (on a complex contour in $k$ we consider the case $\sigma=+1$ in what follows), one finds (this Green's function differs from the $t$-retarded Green's function, constructed on a complex contour in $k^{0}$ )

$$
G(x, \tau)=\frac{2 \theta(\tau)}{(2 \pi)^{3}} \begin{cases}\frac{\tan ^{-1}\left(\sqrt{-x^{2}-\tau^{2}} / \tau\right)}{\left(-x^{2}-\tau^{2}\right)^{3 / 2}}-\frac{\tau}{x^{2}\left(x^{2}+\tau^{2}\right)}, & x^{2}+\tau^{2}<0, \\ \frac{1}{2} \frac{1}{\left(x^{2}+\tau^{2}\right)^{3 / 2}} \ln \left|\frac{\tau-\sqrt{\tau^{2}+x^{2}}}{\tau+\sqrt{\tau^{2}+x^{2}}}\right|-\frac{\tau}{x^{2}\left(x^{2}+\tau^{2}\right)}, & x^{2}+\tau^{2}>0,\end{cases}
$$

where we have written $x^{2} \equiv x^{\mu} x_{\mu}$. 
With the help of this Green's function, the solutions of (3.3) for the self-fields (substituting the current from (2.11)) are

$$
\begin{aligned}
a_{\mathrm{self}}^{\mu}(x, \tau) & =\frac{e_{0}}{\lambda} \int d^{4} x^{\prime} d \tau^{\prime} G\left(x-x^{\prime}, \tau-\tau^{\prime}\right) \dot{x}^{\mu}\left(\tau^{\prime}\right) \delta^{4}\left(x^{\prime}-x\left(\tau^{\prime}\right)\right) \\
& =\frac{e_{0}}{\lambda} \int d \tau^{\prime} \dot{x}^{\mu}\left(\tau^{\prime}\right) G\left(x-x\left(\tau^{\prime}\right), \tau-\tau^{\prime}\right), \\
a_{\text {self }}^{5}(x, \tau) & =\frac{e_{0}}{\lambda} \int d^{4} x^{\prime} d \tau^{\prime} G\left(x-x\left(\tau^{\prime}\right), \tau-\tau^{\prime}\right) .
\end{aligned}
$$

The Green's function is written as a scalar, acting in the same way on all five components of the source $j^{\alpha}$; to assure that the resulting field is in Lorentz gauge, however, it should be written as a five by five matrix, with the factor $\delta_{\beta}^{\alpha}-k^{\alpha} k_{\beta} / k^{2}\left(k_{5}=k\right)$ included in the integrand. Since here we compute only the gauge-invariant field strengths, this extra term will not influence any of the results. It then follows that the generalized Lorentz force for the self-action (the force of the fields generated by the world line on a point $x^{\mu}(\tau)$ of the trajectory), along with the effect of external fields, is

$$
\begin{aligned}
M \ddot{x}^{\mu}= & \left.\frac{e_{0}^{2}}{\lambda} \int d \tau^{\prime}\left(\dot{x}^{\nu}(\tau) \dot{x}_{v}\left(\tau^{\prime}\right) \partial^{\mu}-\dot{x}^{\nu}(\tau) \dot{x}^{\mu}\left(\tau^{\prime}\right) \partial_{v}\right) G\left(x-x\left(\tau^{\prime}\right)\right)\right|_{x=x(\tau)} \\
& +\left.\frac{e_{0}^{2}}{\lambda} \int d \tau^{\prime}\left(\partial^{\mu}-\dot{x}^{\mu}\left(\tau^{\prime}\right) \partial_{\tau}\right) G\left(x-x\left(\tau^{\prime}\right)\right)\right|_{x=x(\tau)}+e_{0}\left(f_{\mathrm{ext} v}^{\mu} \dot{x}^{\nu}+f_{\mathrm{ext} 5}^{\mu}\right) .
\end{aligned}
$$

We define $u \equiv\left(x_{\mu}(\tau)-x_{\mu}\left(\tau^{\prime}\right)\right)\left(x^{\mu}(\tau)-x^{\mu}\left(\tau^{\prime}\right)\right)$, so that

$$
\partial_{\mu}=2\left(x_{\mu}(\tau)-x_{\mu}\left(\tau^{\prime}\right)\right) \frac{\partial}{\partial u}
$$

Equation (3.7) then becomes

$$
\begin{aligned}
M \ddot{x}^{\mu}= & 2 \frac{e_{0}^{2}}{\lambda} \int d \tau^{\prime}\left[\dot{x}^{v}(\tau) \dot{x}_{v}\left(\tau^{\prime}\right)\left(x^{\mu}(\tau)-x^{\mu}\left(\tau^{\prime}\right)\right)\right. \\
& \left.-\dot{x}^{v}(\tau) \dot{x}^{\mu}\left(\tau^{\prime}\right)\left(x_{v}(\tau)-x_{v}\left(\tau^{\prime}\right)\right)\right]\left.\frac{\partial}{\partial u} G\left(x-x\left(\tau^{\prime}\right)\right)\right|_{x=x(\tau)} \\
& +\left.\frac{e_{0}^{2}}{\lambda} \int d \tau^{\prime}\left[2\left(x^{\mu}(\tau)-x^{\mu}\left(\tau^{\prime}\right)\right) \frac{\partial}{\partial u}-\dot{x}^{\mu}\left(\tau^{\prime}\right) \partial_{\tau}\right] G\left(x-x\left(\tau^{\prime}\right), \tau-\tau^{\prime}\right)\right|_{x=x(\tau)} \\
+ & e_{0}\left(f_{\mathrm{ext} v}^{\mu} \dot{x}^{\nu}+f_{\mathrm{ext} 5}^{\mu}\right) .
\end{aligned}
$$

In the self-interaction problem where $\tau \rightarrow \tau^{\prime}, x^{\mu}\left(\tau^{\prime}\right)-x^{\mu}(\tau) \rightarrow 0$, Green's function is very divergent. Therefore one can expand all expressions in $\tau^{\prime \prime}=\tau-\tau^{\prime}$ assuming that the dominant contribution is from the neighborhood of small $\tau^{\prime \prime}$. The divergent terms are later 
absorbed into the mass and charge definitions leading to renormalization (effective mass and charge). Expanding the integrands in Taylor series around the most singular point $\tau=\tau^{\prime}$ and keeping the lowest-order terms, the variable $u$ reduces to

$$
u \cong \dot{x}^{\mu} \dot{x}_{\mu} \tau^{\prime \prime 2}-\dot{x}^{\mu} \ddot{x}_{\mu} \tau^{\prime \prime 3}+\frac{1}{3} \dot{x}^{\mu} \dddot{x}_{\mu} \tau^{\prime \prime 4}+\frac{1}{4} \ddot{x}^{\mu} \ddot{x}_{\mu} \tau^{\prime \prime 4}
$$

We now recall the definition of the off-shell deviation $\varepsilon$ given in (2.20), along with its derivatives:

$$
\begin{gathered}
\dot{x}^{\mu} \dot{x}_{\mu}=-1+\varepsilon, \\
\dot{x}^{\mu} \ddot{x}_{\mu}=\frac{1}{2} \dot{\varepsilon}, \\
\dot{x}^{\mu} \dddot{x}_{\mu}+\ddot{x}^{\mu} \ddot{x}_{\mu}=\frac{1}{2} \ddot{\varepsilon} .
\end{gathered}
$$

Next, we define

$$
\begin{aligned}
& \omega \equiv \frac{1}{12} \dot{x}_{\mu} \dddot{x}^{\mu}+\frac{1}{8} \ddot{\varepsilon}, \\
& \Delta \equiv-\frac{1}{2} \dot{\varepsilon} \tau^{\prime \prime}+\omega \tau^{\prime \prime 2} .
\end{aligned}
$$

Using these definitions along with those of (3.10) and (3.11), we find

$$
\frac{u+\tau^{2}}{\tau^{\prime \prime 2}} \cong \varepsilon+\Delta
$$

We then expand Green's function to leading orders:

$$
\begin{aligned}
\frac{\partial G}{\partial u} \cong & \frac{\Theta\left(\tau^{\prime \prime}\right) f_{1}(\varepsilon+\Delta)}{(2 \pi)^{3} \tau^{\prime \prime 5}}=\frac{\Theta\left(\tau^{\prime \prime}\right)}{(2 \pi)^{3}}\left[\frac{f_{1}(\varepsilon)}{\tau^{\prime \prime 5}}-\frac{\dot{\varepsilon} f_{1}^{\prime}(\varepsilon)}{2 \tau^{\prime \prime 4}}+\left(\omega f_{1}^{\prime}(\varepsilon)+\frac{1}{8} \dot{\varepsilon}^{2} f_{1}^{\prime \prime}(\varepsilon)\right) \frac{1}{\tau^{\prime \prime}}\right] \\
\frac{\partial G}{\partial \tau^{\prime \prime}} \cong & \frac{2 \Theta\left(\tau^{\prime \prime}\right) f_{2}(\varepsilon+\Delta)}{(2 \pi)^{3} \tau^{\prime \prime 4}}+\frac{2 \delta\left(\tau^{\prime \prime}\right) f_{3}(\varepsilon+\Delta)}{(2 \pi)^{3} \tau^{\prime \prime 3}} \\
= & \frac{2 \Theta\left(\tau^{\prime \prime}\right)}{(2 \pi)^{3}}\left[\frac{f_{2}(\varepsilon)}{\tau^{\prime \prime 4}}-\frac{\dot{\varepsilon} f_{2}^{\prime}(\varepsilon)}{2 \tau^{\prime \prime 3}}+\left(\omega f_{2}^{\prime}(\varepsilon)+\frac{1}{8} \dot{\varepsilon}^{2} f_{2}^{\prime \prime}(\varepsilon)\right) \frac{1}{\tau^{\prime \prime 2}}\right] \\
& +\frac{2 \delta\left(\tau^{\prime \prime}\right)}{(2 \pi)^{3}}\left[\frac{f_{3}(\varepsilon)}{\tau^{\prime \prime 3}}-\frac{\dot{\varepsilon} f_{3}^{\prime}(\varepsilon)}{2 \tau^{\prime \prime 2}}+\left(\omega f_{3}^{\prime}(\varepsilon)+\frac{1}{8} \dot{\varepsilon}^{2} f_{3}^{\prime \prime}(\varepsilon)\right) \frac{1}{\tau^{\prime \prime}}\right]
\end{aligned}
$$


where $f^{\prime} \equiv d f / d \varepsilon$. For $\varepsilon<0$

$$
\begin{aligned}
& f_{1}(\varepsilon)=\frac{3 \tan ^{-1}(\sqrt{-\varepsilon})}{(-\varepsilon)^{5 / 2}}-\frac{3}{\varepsilon^{2}(1-\varepsilon)}+\frac{2}{\varepsilon(1-\varepsilon)^{2}}, \\
& f_{2}(\varepsilon)=\frac{3 \tan ^{-1}(\sqrt{-\varepsilon})}{(-\varepsilon)^{5 / 2}}-\frac{1}{\varepsilon^{2}}-\frac{2-\varepsilon}{\varepsilon^{2}(1-\varepsilon)}, \\
& f_{3}(\varepsilon)=\frac{\tan ^{-1}(\sqrt{-\varepsilon})}{(-\varepsilon)^{3 / 2}}+\frac{1}{\varepsilon(1-\varepsilon)}
\end{aligned}
$$

and for $\varepsilon>0$

$$
\begin{aligned}
& f_{1}(\varepsilon)=\frac{(3 / 2) \ln |(1+\sqrt{\varepsilon}) /(1-\sqrt{\varepsilon})|}{\varepsilon^{5 / 2}}-\frac{3}{\varepsilon^{2}(1-\varepsilon)}+\frac{2}{\varepsilon(1-\varepsilon)^{2}}, \\
& f_{2}(\varepsilon)=\frac{(3 / 2) \ln |(1+\sqrt{\varepsilon}) /(1-\sqrt{\varepsilon})|}{\varepsilon^{5 / 2}}-\frac{1}{\varepsilon^{2}}-\frac{2-\varepsilon}{\varepsilon^{2}(1-\varepsilon)} \\
& f_{3}(\varepsilon)=-\frac{(1 / 2) \ln |(1+\sqrt{\varepsilon}) /(1-\sqrt{\varepsilon})|}{\varepsilon^{3 / 2}}+\frac{1}{\varepsilon(1-\varepsilon)} .
\end{aligned}
$$

For either sign of $\varepsilon$, when $\varepsilon \approx 0$,

$$
\begin{aligned}
& f_{1}(\varepsilon) \approx \frac{8}{5}+\frac{24}{7} \varepsilon+\frac{16}{3} \varepsilon^{2}+O\left(\varepsilon^{3}\right), \\
& f_{2}(\varepsilon) \approx-\frac{2}{5}-\frac{4}{7} \varepsilon-\frac{2}{3} \varepsilon^{2}+O\left(\varepsilon^{3}\right), \\
& f_{3}(\varepsilon) \approx \frac{2}{3}+\frac{4}{5} \varepsilon+\frac{6}{7} \varepsilon^{2}+O\left(\varepsilon^{3}\right) .
\end{aligned}
$$

One sees that the derivatives in (3.14) have no singularity in $\varepsilon$ at $\varepsilon=0$.

From (3.6), we have

$$
\begin{aligned}
f_{\text {self } 5}^{\mu}(x(\tau), \tau)= & e \int\left[2\left(x^{\mu}(\tau)-x^{\mu}\left(\tau^{\prime}\right)\right) \frac{\partial}{\partial u}-\dot{x}^{\mu}\left(\tau^{\prime}\right) \partial_{\tau}\right] \\
& \times\left. G\left(x-x\left(\tau^{\prime}\right), \tau-\tau^{\prime}\right)\right|_{x=x(\tau)} d \tau^{\prime} .
\end{aligned}
$$

We then expand $x^{\mu}(\tau)-x^{\mu}\left(\tau^{\prime}\right)$ and $\dot{x}^{\mu}(\tau)-\dot{x}^{\mu}\left(\tau^{\prime}\right)$ in power series in $\tau^{\prime \prime}$ and write the integrals formally with infinite limits. 
Substituting (3.18) into (3.2), we obtain (note that $x^{\mu}$ and its derivatives are evaluated at the point $\tau$ and are not subject to the $\tau^{\prime \prime}$ integration), after integrating by parts using $\delta\left(\tau^{\prime \prime}\right)=$ $\left(\partial / \partial \tau^{\prime \prime}\right) \theta\left(\tau^{\prime \prime}\right)$,

$$
\begin{aligned}
\frac{M}{2} \dot{\varepsilon}=\frac{2 e_{0}^{2}}{\lambda(2 \pi)^{3}} \int_{-\infty}^{\infty} d \tau^{\prime \prime} & {\left[\frac{g_{1}}{\tau^{\prime \prime 4}}(\varepsilon-1)-\frac{g_{2}}{\tau^{\prime \prime 3}} \frac{\dot{\varepsilon}}{2}+\frac{g_{3}}{\tau^{\prime \prime 2}} \dot{x}_{v} \dddot{x}^{\nu}\right.} \\
& -\frac{h_{1}}{\tau^{\prime \prime 3}}(\varepsilon-1) \dot{\varepsilon}+\frac{h_{2}}{2 \tau^{\prime \prime 2}} \dot{\varepsilon}^{2} \\
& \left.+\frac{h_{3}}{\tau^{\prime \prime 2}}(\varepsilon-1) \omega+\frac{h_{4}}{8 \tau^{\prime \prime 2}} \dot{\varepsilon}^{2}(\varepsilon-1)\right] \Theta\left(\tau^{\prime \prime}\right)+e_{0} \dot{x}_{\mu} f_{\mathrm{ext} 5}^{\mu}
\end{aligned}
$$

where we have defined

$$
\begin{gathered}
g_{1}=f_{1}-f_{2}-3 f_{3}, \quad g_{2}=\frac{1}{2} f_{1}-f_{2}-2 f_{3}, \quad g_{3}=\frac{1}{6} f_{1}-\frac{1}{2} f_{2}-\frac{1}{2} f_{3}, \\
h_{1}=\frac{1}{2} f_{1}^{\prime}-\frac{1}{2} f_{2}^{\prime}-f_{3}^{\prime}, \quad h_{2}=\frac{1}{4} f_{1}^{\prime}-\frac{1}{2} f_{2}^{\prime}-\frac{1}{2} f_{3}^{\prime}, \quad h_{3}=\left(f_{1}^{\prime}-f_{2}^{\prime}-f_{3}^{\prime}\right), \\
h_{4}=f_{1}^{\prime \prime}-f_{2}^{\prime \prime}-f_{3}^{\prime \prime} .
\end{gathered}
$$

The integrals are divergent at the lower bound $\tau^{\prime \prime}=0$ imposed by the $\theta$-function; we therefore take these integrals to a cut-off $\mu>0$. Equation (3.19) then becomes

$$
\begin{aligned}
\frac{M}{2} \dot{\varepsilon}=\frac{2 e_{0}^{2}}{\lambda(2 \pi)^{3}}[ & \frac{g_{1}}{3 \mu^{3}}(\varepsilon-1)-\frac{g_{2}}{4 \mu^{2}} \dot{\varepsilon}+\frac{g_{3}}{\mu} \dot{x}_{v} \dddot{x}^{\nu} \\
& \left.-\frac{h_{1}}{2 \mu^{2}}(\varepsilon-1) \dot{\varepsilon}+\frac{h_{2}}{2 \mu} \dot{\varepsilon}^{2}+\frac{h_{3}}{\mu}(\varepsilon-1) \omega+\frac{h_{4}}{8 \mu} \dot{\varepsilon}^{2}(\varepsilon-1)\right]+e_{0} \dot{x}_{\mu} f_{\mathrm{ext} 5}^{\mu} .
\end{aligned}
$$

Following a similar procedure, we obtain from(3.7)

$$
\begin{aligned}
M \ddot{x}^{\mu}=\frac{2 e_{0}^{2}}{\lambda(2 \pi)^{3}}[ & -\frac{1}{2}\left((1-\varepsilon) \ddot{x}^{\mu}+\frac{\dot{\varepsilon}}{2} \dot{x}^{\mu}\right)\left(\frac{f_{1}}{2 \mu^{2}}-\frac{\dot{\varepsilon} f_{1}^{\prime}}{2 \mu}\right)+\frac{f_{1}}{3 \mu}\left(\dot{x}_{v} \dddot{x}^{v} \dot{x}^{\mu}+(1-\varepsilon) \dddot{x}^{\mu}\right) \\
& \left.+\frac{g_{1}}{3 \mu^{3}} \dot{x}^{\mu}-\frac{g_{2}}{2 \mu^{2}} \ddot{x}^{\mu}+\frac{g_{3}}{\mu} \dddot{x}^{\mu}-\frac{h_{1} \dot{\varepsilon}}{2 \mu^{2}} \dot{x}^{\mu}+\frac{h_{2} \dot{\varepsilon}}{\mu} \ddot{x}^{\mu}+\frac{h_{3} \omega}{\mu} \dot{x}^{\mu}+\frac{h_{4} \dot{\varepsilon}^{2}}{8 \mu} \dot{x}^{\mu}\right] \\
& +e_{0}\left(f_{\mathrm{ext} v}^{\mu} \dot{x}^{\nu}+f_{\mathrm{ext} 5}^{\mu}\right) .
\end{aligned}
$$



find

Substituting (3.21) for the coefficients of the $\dot{x}^{\mu}$ terms in the second line of (3.22), we

$$
\begin{aligned}
M(\varepsilon, \dot{\varepsilon}) \ddot{x}^{\mu}= & -\frac{1}{2} \frac{M(\varepsilon, \dot{\varepsilon})}{(1-\varepsilon)} \dot{\varepsilon} \dot{x}^{\mu}+\frac{2 e_{0}^{2}}{\lambda(2 \pi)^{3} \mu} F(\varepsilon)\left[\dddot{x}^{\mu}+\frac{1}{(1-\varepsilon)} \dot{x}_{v} \dddot{x}^{\nu} \dot{x}^{\mu}\right] \\
& +\frac{e_{0} \dot{x}^{\mu} \dot{x}_{v} f_{\text {ext } 5}^{v}}{1-\varepsilon}+e_{0} f_{\text {ext } v}^{\mu} \dot{x}^{\nu}+e_{0} f_{\text {ext } 5}^{\mu}
\end{aligned}
$$

where

$$
F(\varepsilon)=\frac{f_{1}}{3}(1-\varepsilon)+g_{3}
$$

Here, the coefficients of $\ddot{x}^{\mu}$ have been grouped formally into a renormalized (off-shell) mass term, defined (as done in the standard radiation reaction problem) as

$$
M(\varepsilon, \dot{\varepsilon})=M+\frac{e^{\prime 2}}{2 \mu}\left[\frac{f_{1}(1-\varepsilon)}{2}+g_{2}\right]-e^{\prime 2}\left[\frac{1}{4} f_{1}^{\prime}(1-\varepsilon)+h_{2}\right] \dot{\varepsilon} .
$$

We have here a Lorentz effective charge as

$$
e^{\prime 2}=\frac{2 e_{0}^{2}}{\lambda(2 \pi)^{3} \mu}
$$

corresponding to a renormalization depending as well on the cut-off. We will call this quantity $e$ in the following formulas, since, in this context, it can be identified with the measured electric charge through the radiation reaction formulas.

We remark that one can change variables, with the help of (2.20) (here, for simplicity, assuming $\varepsilon<1$ ), to obtain a differential equation in which all derivatives with respect to $\tau$ are replaced by derivatives with respect to the proper time $s$. The coefficient of the second derivative of $x^{\mu}$ with respect to $s$, as well as "effective mass" for the proper-time equation, is then given by

$$
M_{S}(\varepsilon, \dot{\varepsilon})=\frac{2}{3 F(\varepsilon)(1-\varepsilon)}\left[M+\frac{e^{2}}{2 c^{3} \mu}\left[f_{1}(1-\varepsilon) 2+g_{2}\right]-\frac{e^{2}}{c^{3}}\left[\frac{1}{4} f_{1}(1-\varepsilon)+h_{2}+\frac{3}{2} F(\varepsilon) \sqrt{1-\varepsilon}\right] \dot{\varepsilon}\right] .
$$

Note that the renormalized mass depends on $\varepsilon(\tau)$; for this quantity to act as a mass, $\varepsilon$ must be slowly varying on some interval on the orbit of the evolution compared to all other motions. The computer analysis we give below indeed shows that there are large intervals of almost constant $\varepsilon$. In case, as at some points, $\varepsilon$ may be rapidly varying, one may consider the definition (3.25) as formal; clearly, however, if $M(\varepsilon, \dot{\varepsilon})$ is large, $\ddot{x}^{\mu}$ will be suppressed (e.g., 
for $\varepsilon$ close to unity, where $M_{S}(\varepsilon, \dot{\varepsilon})$ goes as $\dot{\varepsilon} /(1-\varepsilon)^{2}$ and $M(\varepsilon, \dot{\varepsilon})$ as $\dot{\varepsilon} /(1-\varepsilon)^{3}$; note that $F(\varepsilon)$ goes as $\left.(1-\varepsilon)^{-3}\right)$.

We now obtain from (3.23)

$$
\begin{aligned}
M(\varepsilon, \dot{\varepsilon}) \ddot{x}^{\mu}= & -\frac{1}{2} \frac{M(\varepsilon, \dot{\varepsilon})}{(1-\varepsilon)} \dot{\varepsilon} \dot{x}^{\mu}+F(\varepsilon) e^{2}\left[\dddot{x}^{\mu}+\frac{1}{(1-\varepsilon)} \dot{x}_{v} \dddot{x}^{v} \dot{x}^{\mu}\right] \\
& +e_{0} f_{\mathrm{ext} v}^{\mu} \dot{x}^{v}+e_{0}\left(\frac{\dot{x}^{\mu} \dot{x}_{v}}{1-\varepsilon}+\delta_{v}^{\mu}\right) f_{\mathrm{ext} 5}^{v}
\end{aligned}
$$

We remark that when one multiplies this equation by $\dot{x}_{\mu}$, it becomes an identity (all of the terms except for $f_{\text {ext } v}^{\mu} \dot{x}^{v}$ may be grouped to be proportional to $\left.\left(\dot{x}^{\mu} \dot{x}_{v} /(1-\varepsilon)+\delta_{v}^{\mu}\right)\right)$; one must use (3.21) to compute the off-shell mass shift $\varepsilon$ corresponding to the longitudinal degree of freedom in the direction of the four-velocity of the particle. Equation (3.28) determines the motion orthogonal to the four-velocity. Equations (3.21) and (3.28) are the fundamental dynamical equations governing the off-shell orbit.

We remark that as in [19] it can be shown that (3.28) reduces to the ordinary (Abraham-Lorentz-Dirac) radiation reaction formula for small, slowly changing $\varepsilon$ and that no instability, no radiation, and no acceleration of the electron occur when it is on shell. There is therefore no "runaway solution" for the exact mass-shell limit of this theory; the unstable Dirac result is approximate for $\varepsilon$ close to, but not precisely, zero.

\section{The $\varepsilon$ Evolution}

We now derive an equation for the evolution of the off-shell deviation, $\varepsilon$, when the external field is removed. We then use this equation to prove that a fixed mass shell is consistent only if the particle is not accelerating, and therefore no runaway solution occurs. Using the definitions

$$
\begin{gathered}
F_{1}(\varepsilon)=\frac{g_{1}(\varepsilon-1)}{3 \mu^{2}}, \quad F_{2}(\varepsilon)=\frac{g_{2}+2(\varepsilon-1) h_{1}}{4 \mu}, \\
F_{3}(\varepsilon)=g_{3}+\frac{1}{12}(\varepsilon-1) h_{3}, \quad F_{4}(\varepsilon)=\frac{1}{2} h_{2}+\frac{1}{8}(\varepsilon-1) h_{4}, \\
F_{5}(\varepsilon)=\frac{1}{8}(\varepsilon-1) h_{3}
\end{gathered}
$$

in (3.21), in the absence of external fields, we write

$$
\dot{x}_{\mu} \dddot{x}^{\mu}=\frac{1}{F_{3}(\varepsilon)}\left\{\frac{M}{2 e^{2}} \dot{\varepsilon}-F_{1}(\varepsilon)+F_{2}(\varepsilon) \dot{\varepsilon}-F_{4}(\varepsilon) \dot{\varepsilon}^{2}-F_{5}(\varepsilon) \ddot{\varepsilon}\right\} .
$$


Differentiating with respect to $\tau$, we find that

$$
\begin{aligned}
\dot{x}_{\mu} \dddot{x}^{\mu}+\ddot{x}_{\mu} \dddot{x}^{\mu}= & \frac{1}{F_{3}}\left\{F_{2}^{\prime} \dot{\varepsilon}^{2}+\ddot{\varepsilon}\left(\frac{M}{2 e^{2}}+F_{2}\right)-F_{1}^{\prime} \dot{\varepsilon}-F_{4}^{\prime} \dot{\varepsilon}^{3}-\left(2 F_{4}+F_{5}^{\prime}\right) \dot{\varepsilon} \ddot{\varepsilon}-F_{5} \dddot{\varepsilon}\right\} \\
& -\frac{F_{3}^{\prime}}{F_{3}^{2}}\left\{F_{2} \dot{\varepsilon}+\frac{M}{2 e^{2}} \dot{\varepsilon}-F_{1}-F_{4} \dot{\varepsilon}^{2}-F_{5} \ddot{\varepsilon}\right\} \dot{\varepsilon} \\
\equiv & H-\frac{F_{5}}{F_{3}} \dddot{\varepsilon} .
\end{aligned}
$$

Together with (4.4), which is the $\tau$ derivative of the last equation in (3.11), one finds (using (4.3)) that

$$
\dot{x}_{\mu} \dddot{x}^{\mu}+3 \ddot{x}_{\mu} \dddot{x}^{\mu}=\frac{1}{2} \dddot{\varepsilon},
$$

which is the $\tau$ derivative of the last equation in (3.11), one finds using(4.3)

$$
\ddot{x}_{\mu} \dddot{x}^{\mu}=\left(\frac{1}{4}+\frac{F_{5}}{2 F_{3}}\right)-\frac{1}{2} H(\varepsilon, \dot{\varepsilon}, \ddot{\varepsilon}) \text {. }
$$

Multiplying (3.28) by $\ddot{x}_{\mu}$ (with no external fields) and using (4.2) and (4.5), we obtain

$$
\left(1+2 \frac{F_{5}}{F_{3}}\right) \dddot{\varepsilon}-A(\varepsilon) \ddot{\varepsilon}+B(\varepsilon) \dot{\varepsilon}^{2}+C(\varepsilon) \dot{\varepsilon}-D(\varepsilon)+E(\varepsilon) \dot{\varepsilon}^{3}+I(\varepsilon) \dot{\varepsilon} \ddot{\varepsilon}=0,
$$

where

$$
\begin{aligned}
A & =\frac{2}{F_{3}}\left(\frac{M}{2 e^{2}}+F_{2}\right)+\frac{2 M(\varepsilon, \dot{\varepsilon})}{e^{2} F(\varepsilon)}+\frac{4 M(\varepsilon, \dot{\varepsilon}) F_{5}}{F_{3} e^{2} F(\varepsilon)} \\
B & =\frac{2 F_{3}^{\prime}}{F_{3}^{2}}\left(F_{2}+\frac{M}{2 e^{2}}\right)-\frac{2 F_{2}^{\prime}}{F_{3}}+\frac{2}{1-\varepsilon} \frac{1}{F_{3}}\left(\frac{M}{2 e^{2}}+F_{2}\right)-\frac{M(\varepsilon, \dot{\varepsilon})}{e^{2} F(\varepsilon)(1-\varepsilon)}-\frac{4 F_{4} M(\varepsilon, \dot{\varepsilon})}{F_{3} e^{2} F(\varepsilon)} \\
C & =\frac{4 M(\varepsilon, \dot{\varepsilon})}{e^{2} F(\varepsilon) F_{3}}\left(\frac{M}{2 e^{2}}+F_{2}\right)-\frac{2}{F_{3}^{2}} F_{1} F_{3}^{\prime}-\frac{2 F_{1}}{(1-\varepsilon) F_{3}}+\frac{2}{F_{3}} F_{1}^{\prime} \\
D & =\frac{4 M(\varepsilon, \dot{\varepsilon}) F_{1}}{e^{2} F(\varepsilon) F_{3}} \\
E & =-\frac{2 F_{4}}{(1-\varepsilon) F_{3}}-2\left(\frac{F_{4} F_{3}^{\prime}}{F_{3}^{2}}-\frac{F_{4}^{\prime}}{F_{3}}\right) \\
I & =2\left(\frac{F_{5}^{\prime}}{F_{3}}+2 \frac{F_{4}}{F_{3}}-\frac{F_{5} F_{3}^{\prime}}{F_{3}^{2}}\right)-\frac{2 F_{5}}{(1-\varepsilon) F_{3}} .
\end{aligned}
$$

We will call this the autonomous equation. 


\section{The Mass Correction Equation and the Orbit Equation: A Compatibility Check}

One needs to verify that the off-shell mass correction equation (4.6) gives the same results as the four-component orbit equation (3.22).

In the absence of external fields, (3.22) can also be written as

$$
a^{\mu}\left(\dot{x}^{0}, \dot{x}^{1}, \dot{x}^{2}, \dot{x}^{3}, \ddot{x}^{0}, \ddot{x}^{1}, \ddot{x}^{2}, \ddot{x}^{3}\right)=\frac{f_{1}}{3 \mu}\left(\dot{x}_{v} \dddot{x}^{v} \dot{x}^{\mu}+(1-\varepsilon) \dddot{x}^{\mu}\right)+\frac{g_{3}}{\mu} \dddot{x}^{\mu}+\frac{h_{3} \dot{x}_{v} \dddot{x}^{\nu}}{3 \mu} \dot{x}^{\mu},
$$

where

$$
\begin{aligned}
a^{\mu}\left(\dot{x}^{0}, \dot{x}^{1}, \dot{x}^{2}, \dot{x}^{3}, \ddot{x}^{0}, \ddot{x}^{1}, \ddot{x}^{2}, \ddot{x}^{3}\right) \equiv & \frac{M \cdot \lambda(2 \pi)^{3}}{2 e_{0}^{2}} \ddot{x}^{\mu}+\frac{1}{2}\left((1-\varepsilon) \ddot{x}^{\mu} \frac{\dot{\varepsilon}}{2} \dot{x}^{\mu}\right)\left(\frac{f_{1}}{2 \mu^{2}}-\frac{\dot{\varepsilon} f_{1}^{\prime}}{2 \mu}\right) \\
& -\frac{g_{1}}{3 \mu^{3}} \dot{x}^{\mu}+\frac{g_{2}}{2 \mu^{2}} \ddot{x}^{\mu}+\frac{h_{1} \varepsilon}{2 \mu^{2}} \dot{x}^{\mu}-\frac{h_{2} \varepsilon}{\mu} \ddot{x}^{\mu}-\frac{h_{3} \ddot{x}_{v} \ddot{x}^{\nu}}{4 \mu} \dot{x}^{\mu}-\frac{h_{4} \dot{\varepsilon}^{2}}{8 \mu} \dot{x}^{\mu}
\end{aligned}
$$

In order to obtain $\dddot{x}^{\mu}$ as a function of the components of the first and second derivatives, one may treat this system as a nonhomogeneous, linear system:

$$
A \dddot{x}=a,
$$

where $A$ is a $4 \times 4$ matrix.

The solutions for this system, which were calculated using the computer package Mathematica, yield initial values for $\dddot{x}^{\mu}$ for a set of initial numeric values assigned in $\dot{x}^{\mu}$ and $\ddot{x}^{\mu}$.

Differentiating (5.1) with respect to $\tau$, we find

$$
\begin{aligned}
& \dot{a}^{\mu}\left(\dot{x}^{0}, \dot{x}^{1}, \dot{x}^{2}, \dot{x}^{3}, \ddot{x}^{0}, \ddot{x}^{1}, \ddot{x}^{2}, \ddot{x}^{3}, \dddot{x}^{0}, \dddot{x}^{1}, \dddot{x}^{2}, \dddot{x}^{3}\right) \\
& =\frac{f_{1}}{3 \mu}\left(\dot{x}_{v} \dddot{x}^{v} \dot{x}^{\mu}+(1-\varepsilon) \dddot{x}^{\mu}\right)+\frac{g_{3} \dddot{x}^{\mu}+\frac{h_{3} \dot{x}_{v} \dddot{x}^{\nu}}{3 \mu} \dot{x}^{\mu} .}{}
\end{aligned}
$$

The initial values $\dddot{x}^{0}, \dddot{x}^{1}, \dddot{x}^{2}, \dddot{x}^{3}$ of (5.4) are the solutions to (5.1) with initial values for $\dot{x}^{0}, \ldots, \dot{x}^{3}$ and $\ddot{x}^{0}, \ldots, \ddot{x}^{3}$ (of course (5.1) and (5.4) have the same initial values of $\dot{x}^{0}, \ldots, \dot{x}^{3}$ and $\ddot{x}^{0}, \ldots, \ddot{x}^{3}$ ). Equations (5.4) and (5.1) have the same form, and therefore the solutions for $\dddot{x}^{\mu}$ and $\dddot{x}^{\mu}$ also have the same form, with the only difference being in replacing $a^{\mu}(5.2)$ with $\dot{a}^{\mu}\left(\dot{x}^{0}, \dot{x}^{1}, \dot{x}^{2}, \dot{x}^{3}, \ddot{x}^{0}, \ddot{x}^{1}, \ddot{x}^{2}, \ddot{x}^{3}, \dddot{x}^{0}, \dddot{x}^{1}, \dddot{x}^{2}, \dddot{x}^{3}\right)$.

Now, we have to show that (4.4) is satisfied. One has

$$
\dot{x}_{\mu} \dddot{x}^{\mu}+3 \ddot{x}_{\mu} \dddot{x}^{\mu}=\frac{1}{2} \dddot{\varepsilon},
$$

where $\dddot{x}^{\mu}\left(\dot{x}^{0}, \dot{x}^{1}, \dot{x}^{2}, \dot{x}^{3}, \ddot{x}^{0}, \ddot{x}^{1}, \ddot{x}^{2}, \ddot{x}^{3}\right)$ are the solutions of $(5.1), \dddot{x}^{\mu}\left(\dot{x}^{0}, \dot{x}^{1}, \dot{x}^{2}, \dot{x}^{3}, \ddot{x}^{0}, \ddot{x}^{1}\right.$, $\left.\ddot{x}^{2}, \ddot{x}^{3}, \dddot{x}^{0}, \dddot{x}^{1}, \dddot{x}^{2}, \dddot{x}^{3}\right)$ are the solutions of $(5.4)$, and $\dddot{\varepsilon}(\varepsilon, \dot{\varepsilon}, \ddot{\varepsilon})$ is the solution of the autonomous 
Table 1: First set. The parameters: $\mu=3 * 10^{-3} \mathrm{GeV}^{-1}, M \alpha=4.84473 * 10^{5} \mathrm{GeV}^{2}$.

\begin{tabular}{|c|c|c|c|}
\hline \multicolumn{2}{|c|}{$\begin{array}{c}\text { Initial conditions for the orbit } \\
\text { equation }\end{array}$} & \multicolumn{2}{|c|}{$\begin{array}{l}\text { The third and forth derivatives (calculated with the initial } \\
\text { conditions for the orbit equation) }\end{array}$} \\
\hline$\dot{x}_{0}=1.6$ & $\ddot{x}_{0}=15$ & $\dddot{x}_{0}=-394197.84731$ & $\dddot{x}_{0}=5.85464 * 10^{9}$ \\
\hline$\dot{x}_{1}=0.1$ & $\ddot{x}_{1}=10$ & $\dddot{x}_{1}=20879.93019$ & $\dddot{x}_{1}=5.62709 * 10^{8}$ \\
\hline$\dot{x}_{2}=0.3$ & $\ddot{x}_{2}=-13$ & $\dddot{x}_{2}=-153331.92947$ & $\dddot{x}_{2}=7.54373 * 10^{8}$ \\
\hline$\dot{x}_{3}=0.9$ & $\ddot{x}_{3}=-23$ & $\dddot{x}_{3}=379634.21816$ & $\dddot{x}_{3}=6.46893 * 10^{9}$ \\
\hline \multicolumn{4}{|c|}{ The orbit equation result } \\
\hline \multicolumn{4}{|c|}{$\dddot{\varepsilon}=2 \cdot\left(\dot{x}_{\mu} \dddot{x}^{\mu}+3 \ddot{x}_{\mu} \dddot{x}^{\mu}\right)=-1.726245753395 * 10^{9}$} \\
\hline \multicolumn{4}{|c|}{ Initial conditions for the autonomous equation } \\
\hline \multicolumn{4}{|c|}{$\varepsilon=1-\dot{x}_{0}^{2}+\dot{x}_{1}^{2}+\dot{x}_{2}^{2}+\dot{x}_{3}^{2}=-0.65$} \\
\hline \multicolumn{4}{|c|}{$\dot{\varepsilon}=2 \cdot\left(-\dot{x}_{0} \cdot \ddot{x}_{0}+\dot{x}_{1} \cdot \ddot{x}_{1}+\dot{x}_{2} \cdot \ddot{x}_{2}+\dot{x}_{3} \cdot \ddot{x}_{3}\right)=-95.2$} \\
\hline \multicolumn{4}{|c|}{$\ddot{\varepsilon}=2 \cdot\left(-\dot{x}_{0} \cdot \dddot{x}_{0}+\dot{x}_{1} \cdot \dddot{x}_{1}+\dot{x}_{2} \cdot \dddot{x}_{2}+\dot{x}_{3} \cdot \dddot{x}_{3}-\ddot{x}_{0} \cdot \ddot{x}_{0}+\ddot{x}_{1} \cdot \ddot{x}_{1}+\ddot{x}_{2} \cdot \ddot{x}_{2}+\ddot{x}_{3} \cdot \ddot{x}_{3}\right)=1.8581 * 10^{6}$} \\
\hline
\end{tabular}

The autonomous equation result

$\dddot{\varepsilon}=-1.726245753395 * 10^{9}$

Table 2: Second set. The parameters: $\mu=3 * 10^{5} \mathrm{GeV}^{-1} \mathrm{~Hz}, M / \alpha=4.84473 * 10^{-10} \mathrm{GeV}^{2}$.

\begin{tabular}{lccc}
\hline \multicolumn{2}{r}{$\begin{array}{r}\text { Initial conditions for the orbit } \\
\text { equation }\end{array}$} & \multicolumn{2}{c}{$\begin{array}{r}\text { The third and forth derivatives (calculated with the initial } \\
\text { conditions for the orbit equation) }\end{array}$} \\
\hline$\dot{x}_{0}=1.001$ & $\ddot{x}_{0}=1.6476$ & $\dddot{x}_{0}=22.5575$ & $\dddot{x}_{0}=124.9841$ \\
$\dot{x}_{1}=0.1$ & $\dddot{x}_{1}=4$ & $\dddot{x}_{2}=6.2052$ & $\dddot{x}_{1}=58.5639$ \\
$\dot{x}_{2}=0.2$ & $\ddot{x}_{2}=1$ & $\dddot{x}_{3}=10.5736$ & $\dddot{x}_{2}=33.0308$ \\
$\dot{x}_{3}=0.3$ & $\ddot{x}_{3}=2$ & $\dddot{x}_{3}=55.5532$ \\
\hline
\end{tabular}

The orbit equation result

$\dddot{\varepsilon}=2 \cdot\left(\dot{x}_{\mu} \dddot{x}^{\mu}+3 \ddot{x}_{\mu} \dddot{x}^{\mu}\right)=36.272327445316$

Initial conditions for the autonomous equation

$\varepsilon=1-\dot{x}_{0}^{2}+\dot{x}_{1}^{2}+\dot{x}_{2}^{2}+\dot{x}_{3}^{2}=0.13800$

$\dot{\varepsilon}=2 \cdot\left(-\dot{x}_{0} \cdot \ddot{x}_{0}+\dot{x}_{1} \cdot \ddot{x}_{1}+\dot{x}_{2} \cdot \ddot{x}_{2}+\dot{x}_{3} \cdot \ddot{x}_{3}\right)=-0.89850$

$\ddot{\varepsilon}=2 \cdot\left(-\dot{x}_{0} \cdot \dddot{x}_{0}+\dot{x}_{1} \cdot \dddot{x}_{1}+\dot{x}_{2} \cdot \dddot{x}_{2}+\dot{x}_{3} \cdot \dddot{x}_{3}-\ddot{x}_{0} \cdot \ddot{x}_{0}+\ddot{x}_{1} \cdot \ddot{x}_{1}+\ddot{x}_{2} \cdot \ddot{x}_{2}+\ddot{x}_{3} \cdot \ddot{x}_{3}\right)=2.62951$

The autonomous equation result

$\dddot{\varepsilon}=36.272327445316$

equation with initial conditions which are consistent with the ones in (5.1) and (5.4) that satisfy (3.11).

In Tables 1 and 2, we see two sets of parameters and initial conditions which were chosen for the components of $\dot{x}^{\mu}$ and $\ddot{x}^{\mu}$. For each set, the orbit equation yields its results for $\dddot{x}^{\mu}$ and $x^{\mu}$ and the autonomous equation yields its results for $\dddot{\varepsilon}$. In both sets the results satisfy (5.5). 
As a further test we compared trajectories of the orbit equations and the autonomous equation using the Runge-Kutta method.

\subsection{The Numerical Solution Techniques}

We investigated the dynamical behavior of the system using two methods: the first is the completely "computerized" method and the second is the "manual" fourth-order RungeKutta algorithm.

The first method is based upon Mathematica's numerical equation-solving set of commands. This method was only used with the autonomous equation (as the orbit equations are too complicated and thus require a different approach). The second "manual" method was used to numerically solve both the orbit equation and the autonomous equation (also using Mathematica's computation power), as follows.

The four dynamical equations (or orbit equations), (3.22), can be written as a set of eight first-order equations of the form

$$
\begin{gathered}
\frac{d \dot{x}^{0}}{d \tau}=\ddot{x}^{0}, \\
\frac{d \dot{x}^{1}}{d \tau}=\ddot{x}^{1}, \\
\frac{d \dot{x}^{2}}{d \tau}=\ddot{x}^{2}, \\
\frac{d \dot{x}^{3}}{d \tau}=\ddot{x}^{3}, \\
\frac{d \ddot{x}^{0}}{d \tau}=f 0\left(\dot{x}^{0}, \dot{x}^{1}, \dot{x}^{2}, \dot{x}^{3}, \ddot{x}^{0}, \ddot{x}^{1}, \ddot{x}^{2}, \ddot{x}^{3}\right), \\
\frac{d \ddot{x}^{1}}{d \tau}=f 1\left(\dot{x}^{0}, \dot{x}^{1}, \dot{x}^{2}, \dot{x}^{3}, \ddot{x}^{0}, \ddot{x}^{1}, \ddot{x}^{2}, \ddot{x}^{3}\right), \\
\frac{d \ddot{x}^{2}}{d \tau}=f 2\left(\dot{x}^{0}, \dot{x}^{1}, \dot{x}^{2}, \dot{x}^{3}, \ddot{x}^{0}, \ddot{x}^{1}, \ddot{x}^{2}, \ddot{x}^{3}\right), \\
\frac{d \ddot{x}^{3}}{d \tau}=f 3\left(\dot{x}^{0}, \dot{x}^{1}, \dot{x}^{2}, \dot{x}^{3}, \ddot{x}^{0}, \ddot{x}^{1}, \ddot{x}^{2}, \ddot{x}^{3}\right) .
\end{gathered}
$$

We assigned the expressions obtained earlier for the four third-order derivatives $\dddot{x}^{0}-$ $\dddot{x}^{3}$ into the functions $f 0-f 3$, respectively, and used the Runge-Kutta method to calculate the trajectory for the system of equations. The algorithm was programmed in Mathematica.

As for the autonomous equation, (4.6), it can be written as a set of three first-order equations of the form

$$
\begin{gathered}
\frac{d \varepsilon}{d \tau}=\dot{\varepsilon}, \\
\frac{d \dot{\varepsilon}}{d \tau}=\ddot{\varepsilon}, \\
\frac{d \ddot{\varepsilon}}{d \tau}=Q(\varepsilon, \dot{\varepsilon}, \ddot{\varepsilon}),
\end{gathered}
$$




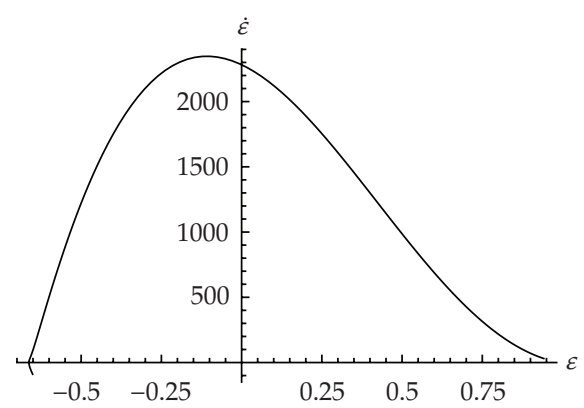

(a)

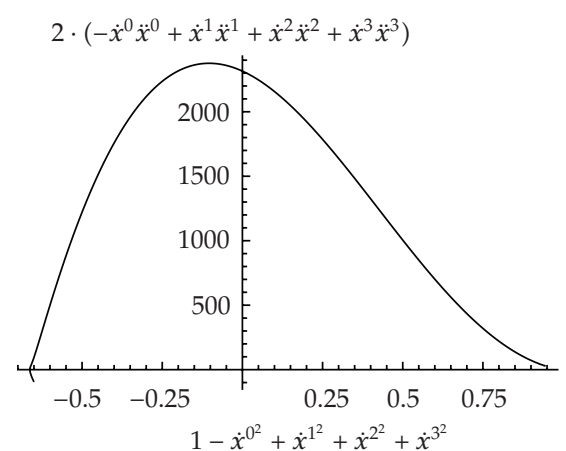

(b)

Figure 1: (a) First set, the autonomous equation trajectory. (b) First set, the orbit equation trajectory.

with

$$
Q=\dddot{\varepsilon}=\frac{1}{1+2\left(F_{5} / F_{3}\right)}\left(A(\varepsilon, \dot{\varepsilon}) \ddot{\varepsilon}-B(\varepsilon, \dot{\varepsilon}) \dot{\varepsilon}^{2}-C(\varepsilon, \dot{\varepsilon}) \dot{\varepsilon}+D(\varepsilon, \dot{\varepsilon})-E(\varepsilon) \dot{\varepsilon}^{3}-I(\varepsilon) \dot{\varepsilon} \ddot{\varepsilon}\right) .
$$

Again, the initial values $\varepsilon, \dot{\varepsilon}, \ddot{\varepsilon}$ for the autonomous quation were chosen to match those of the orbit equation, according to (3.11). Thus, they obey the three conditions

$$
\begin{gathered}
\varepsilon=\dot{x}_{\mu} \dot{x}^{\mu}+1, \\
\dot{\varepsilon}=2 \dot{x}_{\mu} \ddot{x}^{\mu}, \\
\ddot{\varepsilon}=2\left(\dot{x}_{\mu} \dddot{x}^{\mu}+\ddot{x}_{\mu} \ddot{x}^{\mu}\right) .
\end{gathered}
$$

With these initial values inserted, we calculated the trajectory for the autonomous equation using the Runge-Kutta method.

As explained above, we used two sets of initial values, which appear in Tables 1 and 2, and evolve to the trajectories shown in Figures 1 and 2, respectively. Figures 1(a) and 2(a) show the trajectories of the autonomous equation for each set, and Figures 1(b) and 2(b) show those of the orbit equation. The identical results demonstrate the compatibility between the orbit equation and the autonomous equation.

Mathematica's numerical equation-solving commands produced identical results to the Runge-Kutta method for the autonomous equation in both sets of the initial conditions.

\subsection{About the Parameters Used}

The calculation carried out uses the parameters $M / \alpha$ and $\mu$, where $\alpha=\mu e^{2}$ (the orbit equation contains the factor $\alpha$ on the right-hand side, so that $M / \alpha$ is a convenient parameter for the numerical analysis). We chose to work with natural units: $\hbar=c=1$, and so energy has units of $\mathrm{GeV}$, and mass ( $m$ and $M$ ) is also in units of GeV. Length $(\hbar / m c)$ is in units of $\mathrm{GeV}^{-1}$, and time $\left(\hbar / m c^{2}\right)$ is in units of $\mathrm{GeV}^{-1}$ (both proper and universal). The velocity is therefore without units, acceleration has units of $\mathrm{GeV}$, and so on. The cutoff frequency, $\mu$, has units of 


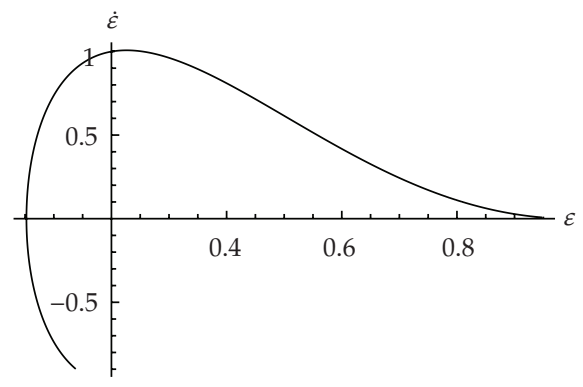

(a)

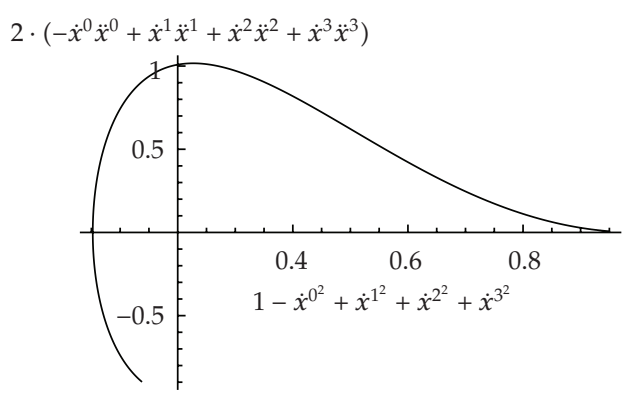

(b)

Figure 2: (a) Second set, the autonomous equation trajectory. (b) Second set, the orbit equation trajectory.

time, $\mathrm{GeV}^{-1}$, and the charge, $e=\sqrt{2 e_{0}^{2} / \lambda(2 \pi)^{3}} \mu$, has no units. The value $e^{2}$ can be identified with the measured electric charge squared. It depends on three bare parameters: $e_{0}, \mu$, and $\lambda$. In our examples we chose these bare parameters so that $e$ would be equal to the electron's charge. The initial conditions were chosen to be close to the mass shell $\left(\varepsilon=1-m^{2} / M^{2} \approx 0\right)$.

\section{The Autonomous Equation and the (Universal Time) Effective Mass $M(\varepsilon, \dot{\varepsilon})$}

In both the orbit equation and the autonomous equation, initial conditions within a basin of attraction will evolve to an orbit of an attractor. In the phase space of $\varepsilon, \dot{\varepsilon}, \ddot{\varepsilon}$, and $\dddot{\varepsilon}$, the orbit of the attractor propagates towards the vicinity of the coordinate $(\varepsilon, \dot{\varepsilon}, \ddot{\varepsilon}, \dddot{\varepsilon})=(1,0,0,0)$, which will simply be named unity.

The nature of the orbit depends on the cutoff frequency $\mu$. Higher cutoff frequency results in a nonfluctuating orbit, while a lower, more physical frequency (closer to zero) results in a fluctuating one. The fluctuations appear as loop cycles in the phase space graphs.

\subsection{Analysis of a Cycling Attractor (with a Lower, More "Physical" Cutoff Frequency)}

In this subsection, we bring an example of a fluctuant attractor's orbit and present its properties. We start with Figures $3(\mathrm{a})$ and $3(\mathrm{~b})$, which are the orbit's phase space graphs $(\varepsilon, \dot{\varepsilon})$ and $(\dot{\varepsilon}, \ddot{\varepsilon})$, respectively.

In Figure 3(c) we show the global Lyapunov exponent of the attractor. We have computed the global Lyapunov exponent by studying the average separation of orbits associated with nearby initial conditions. Segments of relative stability and instability can be observed.

Despite the fluctuations which create temporary instabilities, in general the Lyapunov exponent decays. A time series of the correlation between the attractor and an orbit with an initial coordinate at a distance of 0.00001 (Figure 3(d)) shows the same phenomenon: the distance between the orbits generally decreases, despite the temporary fluctuations. Thus, no evidence of chaotic behavior is found. 


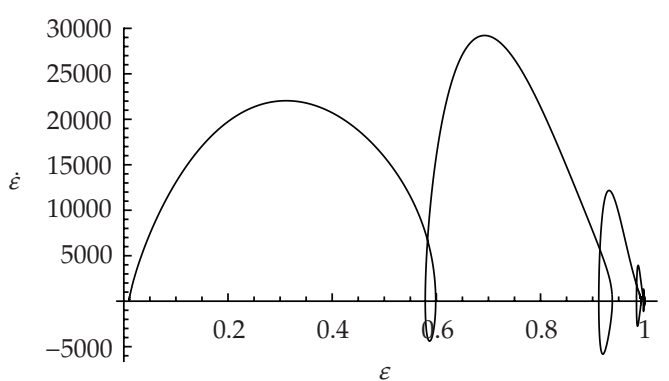

(a)

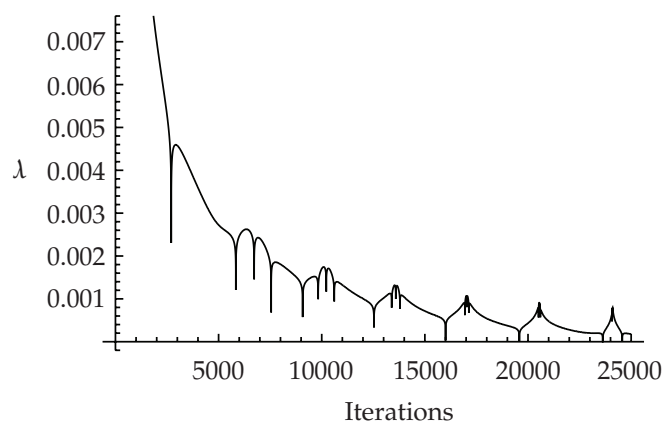

(c)

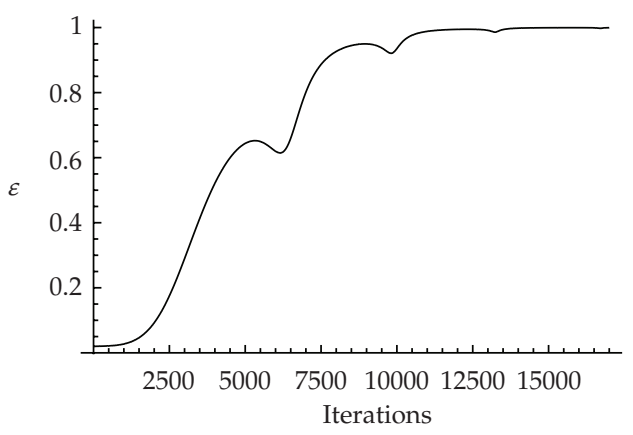

(e)

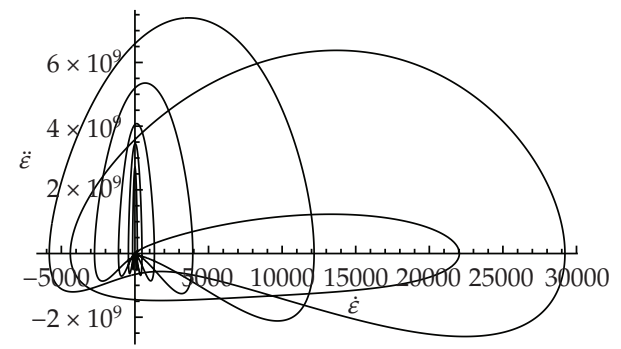

(b)

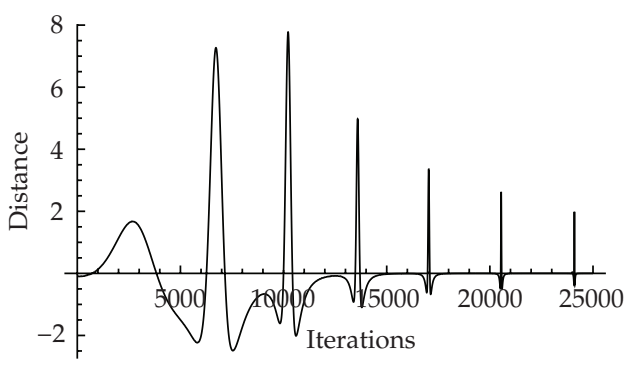

(d)

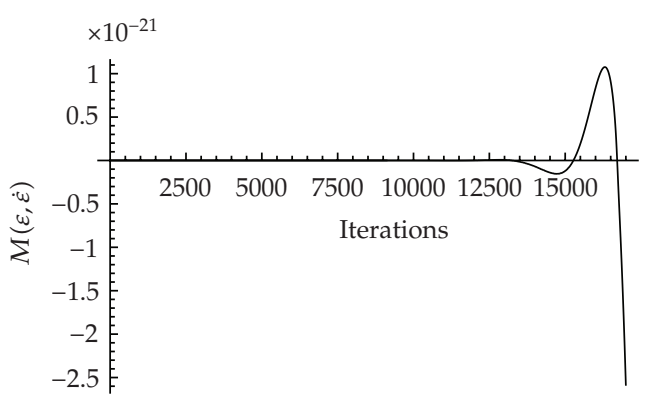

(f)

Figure 3: (a) and (b) The orbit of an attractor in two phase space graphs. Initial conditions: $\varepsilon=0.01, \dot{\varepsilon}=55$, $\ddot{\varepsilon}=10^{6}, M / \alpha=556.85 \mathrm{GeV}^{2}$, and $\mu=10^{-5} \mathrm{GeV}^{-1}$. (c) The Lyapunov exponent $\lambda$. (d) The correlation between two close orbits: the attractor and an orbit which starts close to it (distance 0.00001). One can clearly see the correlation between the orbits, which is not a chaotic pattern. (e) and (f) $\varepsilon$ and the effective mass as a function of the number of time iterations. As $\varepsilon$ becomes close to unity the mass fluctuation amplitude grows significantly.

Figures 3(e) and 3(f) show the attractor's $\varepsilon$ and the effective mass as a function of the universal time iterations. The effective mass fluctuation amplitude grows significantly near unity.

We will analyze three consecutive cycles (fluctuations) in our chosen attractor and observe the phase space trajectory, its stability, and the mass behavior. 


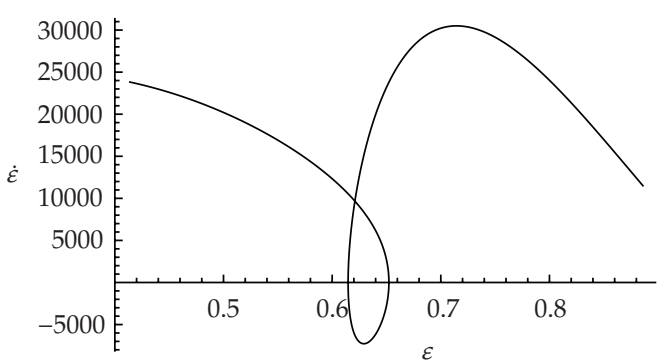

(a)

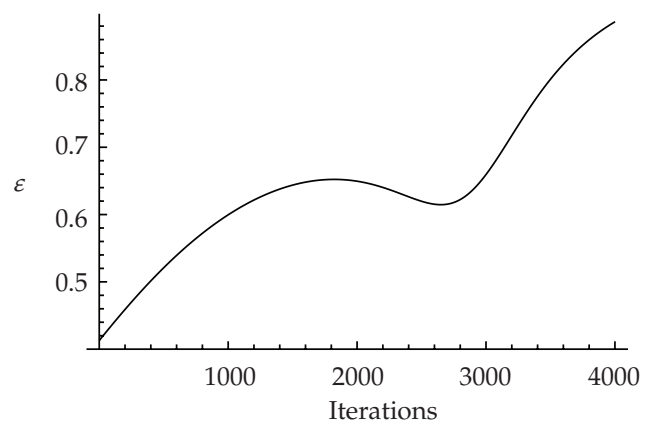

(c)

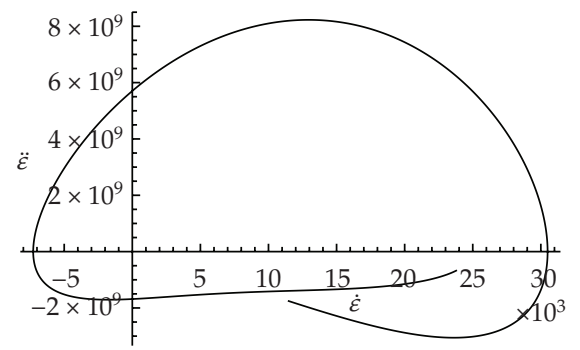

(b)

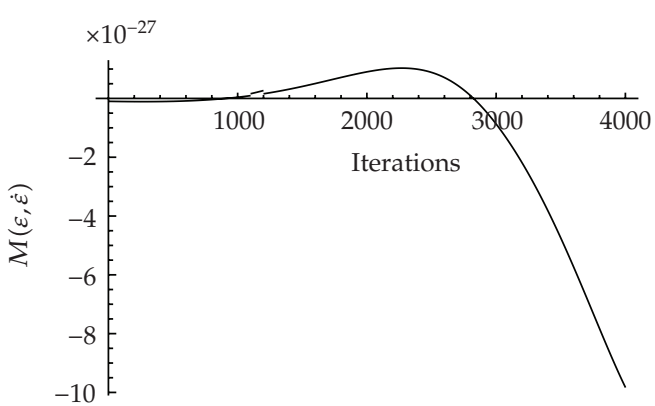

(d)

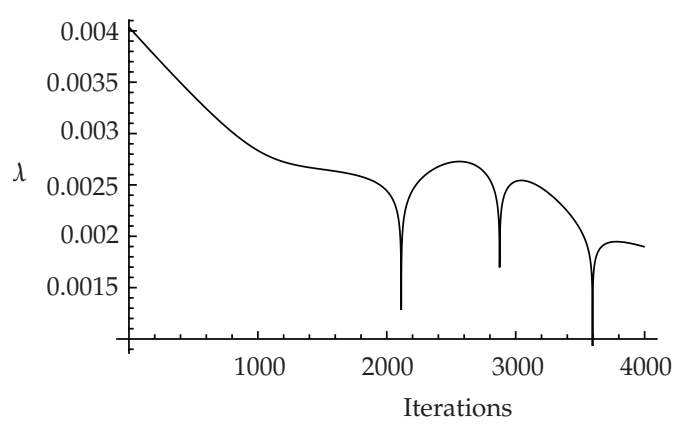

(e)

Figure 4: (a) and (b). The $\varepsilon, \dot{\varepsilon}$ and the $\dot{\varepsilon}, \ddot{\varepsilon}$ graphs, respectively. One can see the loop cycle in both phase space graphs. (c) and (d) $\varepsilon$ and the (universal time) effective mass $M(\varepsilon, \dot{\varepsilon})$ as functions of the number of iterations. One can see how $\varepsilon$ temporarily decreases during the cycle. The effective mass significantly grows (in absolute value) as the cycle terminates. (e) The Lyapunov exponent fluctuates during the cycle (relative instability) but generally decreases.

\subsubsection{First Cycle}

In Figures $4(\mathrm{a})$ and $4(\mathrm{~b})$, we see $\varepsilon, \dot{\varepsilon}$ and $\dot{\varepsilon}, \ddot{\varepsilon}$ graphs of this cycle, respectively. Figures 4(c) and 4(d) show $\varepsilon$ and the effective mass as functions of the number of iterations. Figure 4(e) shows the Lyapunov exponent. The orbit in Figure 4(a) enters a region of folding, which develops to a loop. In Figure 4(b), one can see an apparent attractor inducing motion beginning at the lower convex portion of the orbit which then turns back in a characteristic way to reach the last point visible on this graph. 
The instability in the Lyapunov exponent (Figure 4(e)) is accompanied by a temporary decrease in the $\varepsilon$ graph (Figure 4(c)). This instability is the climax of the loop cycle in the $\varepsilon, \dot{\varepsilon}$ and the $\dot{\varepsilon}, \ddot{\varepsilon}$ graphs. As the loop cycle terminates, $\varepsilon$ climbs to a higher value than the one it had when the cycle began, and the exponent continues to decay.

In Figure 4(d) we see that the effective mass fluctuates with a growing amplitude. As the cycle reaches towards its end, the effective mass continues to a higher amplitude and has its highest absolute values.

\subsubsection{Second Cycle}

The attractor's orbit continues to a second cycle, as can be seen in the $\varepsilon, \dot{\varepsilon}$ and $\dot{\varepsilon}, \ddot{\varepsilon}$ graphs (Figures $5(\mathrm{a})$ and $5(\mathrm{~b})$, resp.). This second cycle is again accompanied by a fluctuation (relative instability) in the Lyapunov exponent, as can be seen in Figure 5(e). The mass, as before, fluctuates with growing amplitude (we can observe the growing values on the $y$-axis in Figure 5(d) in comparison to Figure 4(d)). As the mass reaches a relatively low absolute value, the Lyapunov exponent rises to a relatively high one (the "jump" area of the exponent graph).

\subsubsection{Third Cycle}

The consecutive cycle behaves in the same way as the two previous ones, as can be seen in Figures 6(a)-6(e). The occurrence of the present loop cycle is seen when $\varepsilon$ has a down spike (around the iteration value 2500 in Figure 6(c)). In this area the Lyapunov exponent has an up spike (Figure 6(e)). The effective mass, in contrast to the Lyapunov exponent, decreases during the cycle but rises to a higher amplitude as it ends (Figure 6(d)).

The above results suggest that as long as the electron "survives" the fluctuations (or cycles) and its orbit doesn't run away to infinity, the effective mass will reach higher absolute values in the end of each cycle. The effective mass, in general, fluctuates with increasing amplitude. As can be seen from the Lyapunov exponent graphs, the attractor is relatively unstable during the cycles but regains stability between them. A correlation is clearly observed between a relatively high absolute value of the effective mass and a relatively low value of the Lyapunov exponent (which indicates a relative stability).

We also saw correlation between our attractor and nearby orbits, as seen in the time series associated with them, even in the vicinity of unity (the light cone). This correlation shows the absence of chaotic behavior.

\subsection{A Smooth (Less Physical) Attractor}

In this subsection we give an example of a smooth attractor, as can be seen in Figures 7(a)$7(\mathrm{e})$.

The only change in the initial conditions and parameters between this smooth orbit and the former, fluctuant, one is the cutoff frequency, which has a higher value now: $10^{-1} \mathrm{GeV}^{-1}$ (instead of $10^{-5} \mathrm{GeV}^{-1}$ in the fluctuant case). This orbit has no loop cycles in its phase space graphs. It has, however, a higher life span than the previous, fluctuant attractor, and thus it stays a longer time in the vicinity of unity. Nevertheless, also in this case the orbit will, at some point, run away to infinity.

Although a higher cutoff frequency is less "physical", the advantage of showing an attractor with relatively high cutoff frequency is that the fluctuations are removed, and the 


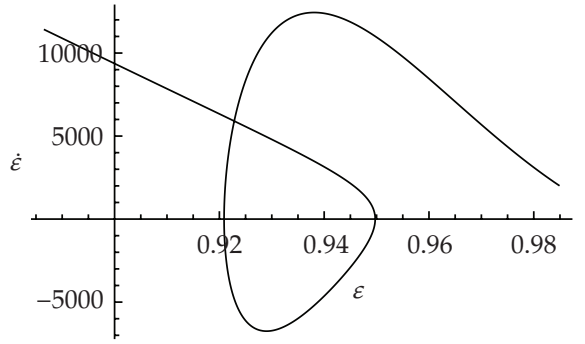

(a)

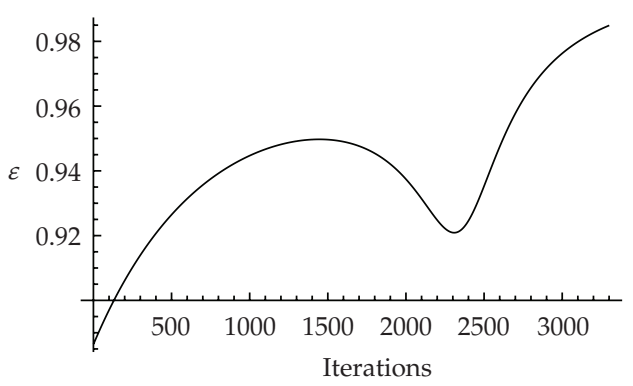

(c)

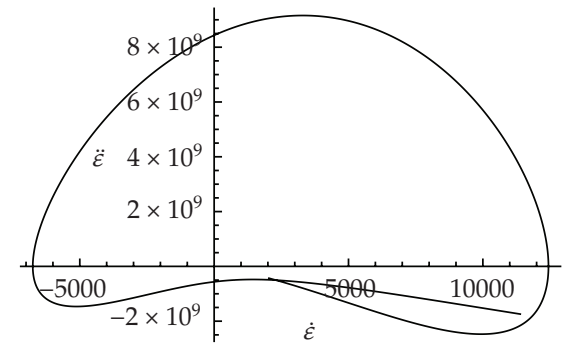

(b)

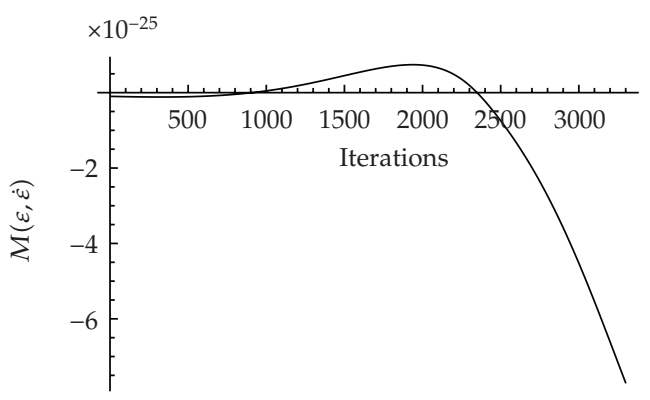

(d)

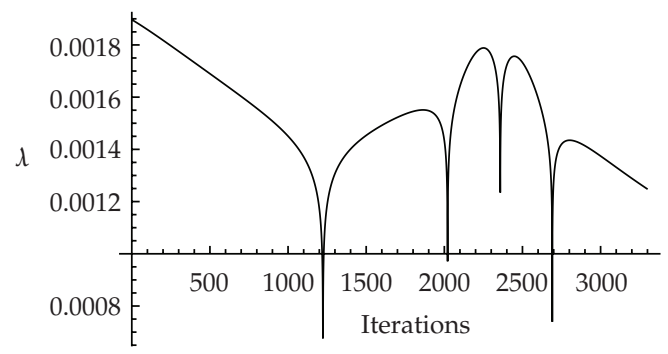

(e)

Figure 5: (a) and (b) The $\varepsilon, \dot{\varepsilon}$ and the $\dot{\varepsilon}, \ddot{\varepsilon}$ phase space graphs, respectively. (c) and (d) $\varepsilon$ and the (universal time) effective mass $M(\varepsilon, \dot{\varepsilon})$ as functions of the number of iterations. As in the previous cycle, the mass fluctuation amplitude grows. (e) Again, the Lyapunov exponent fluctuates during the cycle and returns to decay as it ends.

"pure" correlation between the growing effective mass and the rising stability (in the form of declining and even negative Lyapunov exponent) can be seen. Indeed, the attractor's Lyapunov exponent in Figure 7(c) turns to be unambiguously negative and smooth, which may indicate the existence of a fixed point.

The attractor's $\varepsilon$ and effective mass as functions of the time iterations appear in Figures 7(d) and 7(e), respectively. There are no fluctuations. We can therefore conclude that the pattern is even clearer in the present example: as the attractor is steadily approaching the light cone, the effective mass is steadily growing (in absolute value), without fluctuations, and the Lyapunov exponent is negative and stable. 


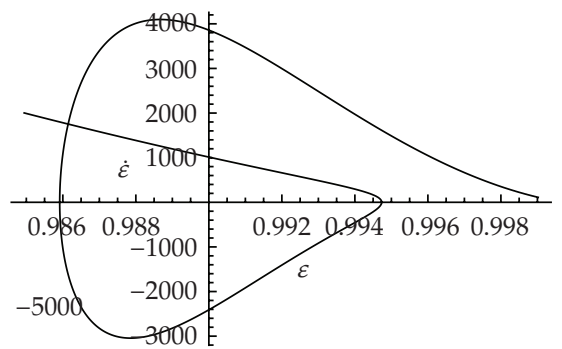

(a)

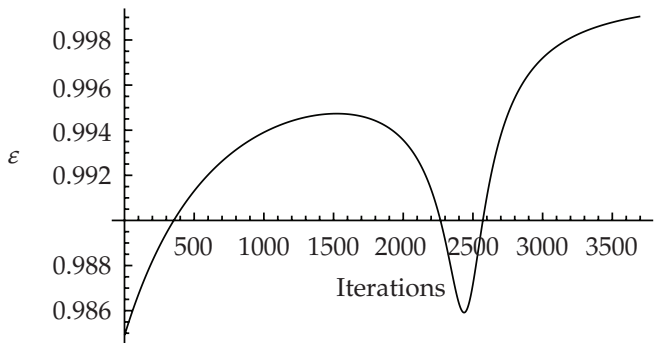

(c)

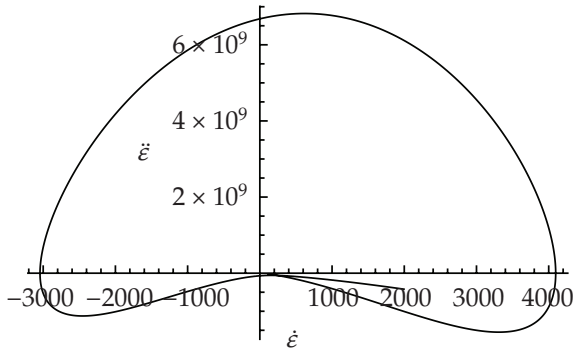

(b)

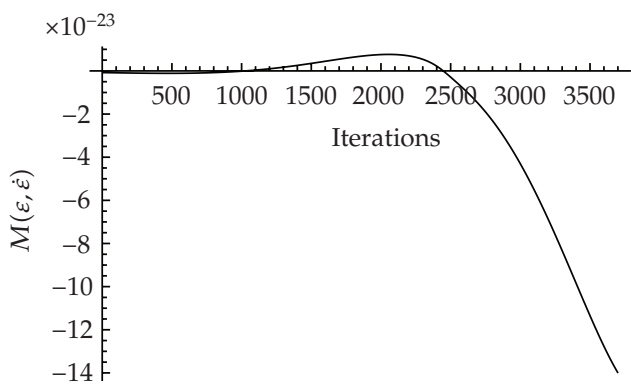

(d)

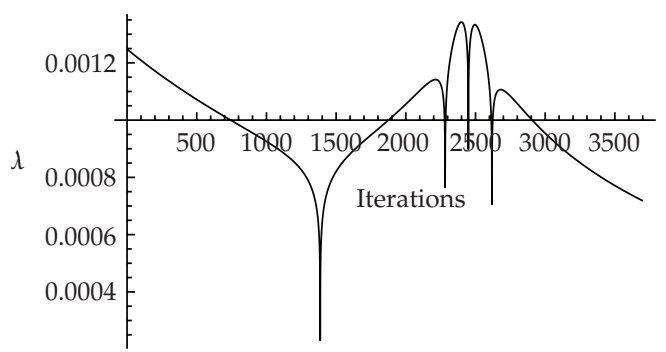

(e)

Figure 6: (a) and (b) The $\varepsilon, \dot{\varepsilon}$ and the $\dot{\varepsilon}, \ddot{\varepsilon}$ phase space graphs, respectively. (c) and (d) $\varepsilon$ and the (universal time) effective mass $M(\varepsilon, \dot{\varepsilon})$ as functions of the number of iterations. As in the previous cycles, the mass fluctuation amplitude significantly grows towards the cycle's end. One should also observe the relatively low value of the effective mass around the iteration value of 2500 (the climax of the cycle). (e) Again, the Lyapunov exponent fluctuates during the cycle and returns to decay afterwards.

\section{The Dynamical Equations}

In this section, we will demonstrate some characteristics the orbit equation exhibits, which were previously found to be properties of the autonomous equation for the off-mass shell.

As a first example, we choose a fluctuant, two-loop-cycle orbit (Figures 8(a)-8(c), 9(a)9(c), 10(a)-10(e)) with given initial conditions, and analyze it. In Figures 8(a)-8(c) we see $\left(\dot{x}^{1}\right.$, $\left.\ddot{x}^{1}\right)$ graphs of the orbit. An attractor can be clearly seen. The orbit reaches velocities which are very large (These velocities, in which $\varepsilon$ is close to unity, correspond to very short segments of the world line due to the factor $\sqrt{1-\varepsilon}$ connecting $d \tau$ and $d s$.) 


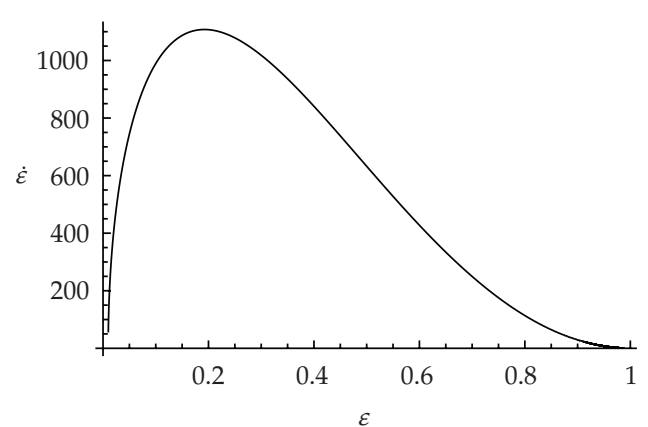

(a)

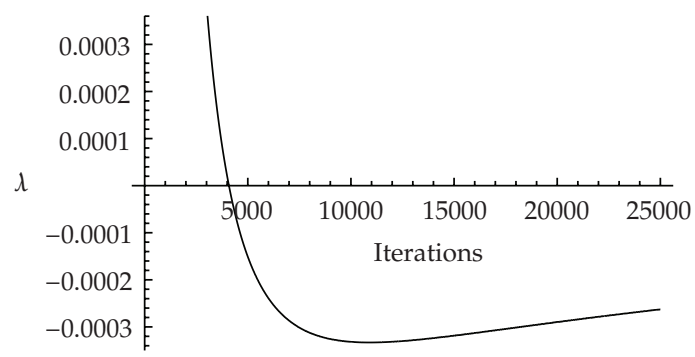

(c)

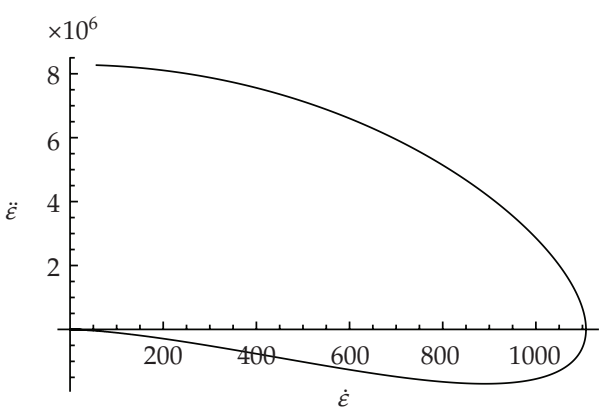

(b)

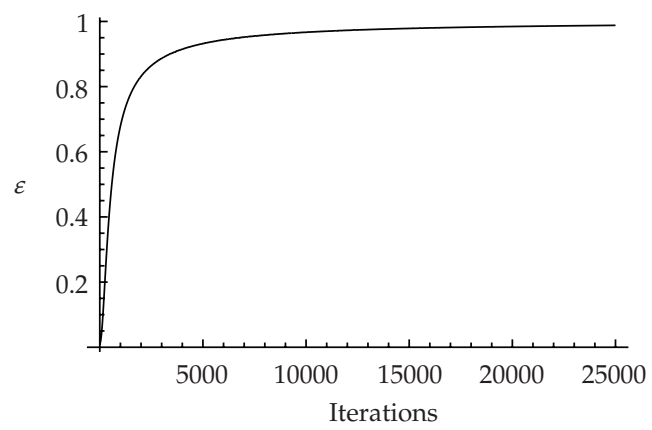

(d)

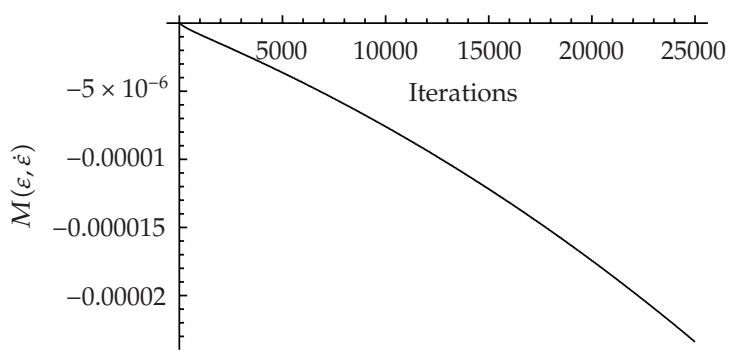

(e)

Figure 7: (a) and (b) An attractor without fluctuations (cycles). (a) $\varepsilon, \dot{\varepsilon}$ graph and (b) $\dot{\varepsilon}, \ddot{\varepsilon}$ graph. (c) Lyapunov exponent and (d) $\varepsilon$ itself, as functions of the (universal) time iterations. (e) The effective mass steadily grows (in absolute value) as $\varepsilon$ moves towards unity.

Figures 9(a)-9(c) show the $(\varepsilon, \dot{\varepsilon})$ graphs of the orbit. While the second loop cycle in the $(\varepsilon, \dot{\varepsilon})$ graph becomes smaller than the first and closer to unity, the second loop cycle in the velocity-acceleration graph becomes larger than the first one.

The fact that the second loop cycle in the velocity-acceleration graph is larger than the first means that during the attraction towards the light cone the velocity reaches more extreme values. One can look at this with connection to the behavior shown in the autonomous equation case, in which each loop cycle causes relative instability.

Figures 10(a)-10(e) show $\varepsilon$, the $\dot{x}^{1}$ component of the velocity, the $\ddot{x}^{1}$ component of the acceleration, the effective mass $M(\varepsilon, \dot{\varepsilon})$, and the Lyapunov exponent, respectively, as 


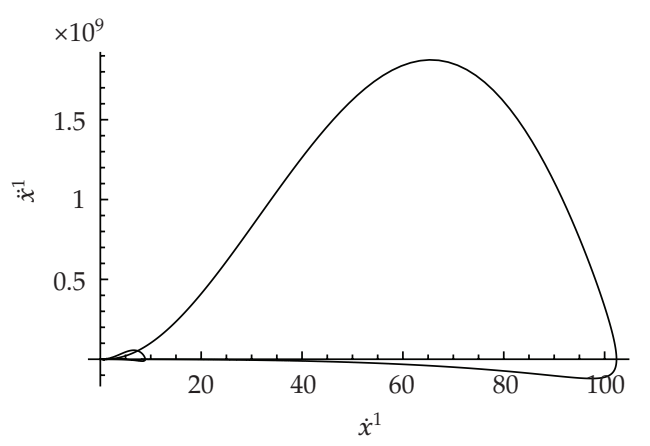

(a)

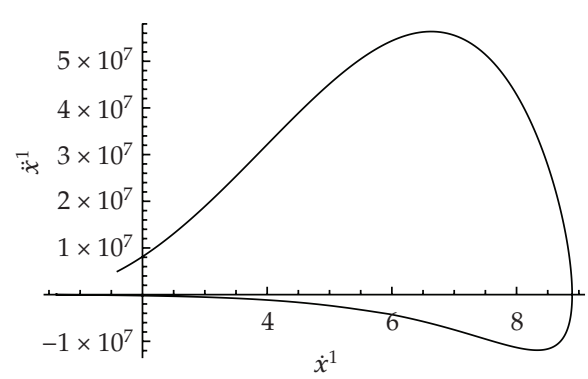

(b)

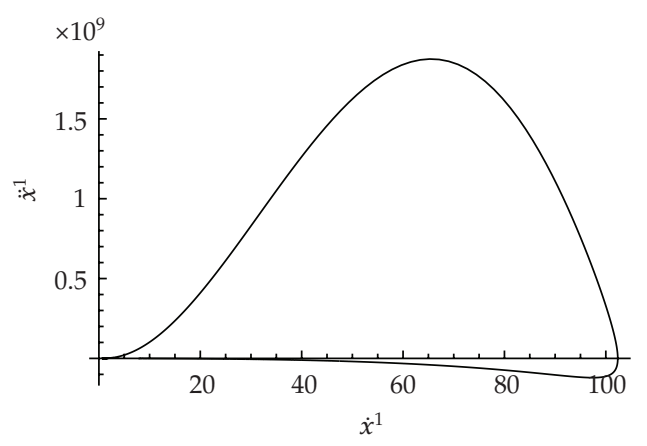

(c)

Figure 8: See also Figure 9. Figures $8(\mathrm{a})$ and 9(a) a two-loop-cycle orbit, as shown in $\dot{x}^{1}, \ddot{x}^{1}$ graph (Figure 8(a)) and $\varepsilon, \dot{\varepsilon}$ graph (Figure 9(a)). Initial conditions: $\dot{x}^{0}=0.8, \dot{x}^{1}=0.1, \dot{x}^{2}=0.1, \dot{x}^{3}=0.1$, $\ddot{x}^{0}=-10000, \ddot{x}^{1}=4, \ddot{x}^{2}=5, \ddot{x}^{3}=7, M / \alpha=4.8 \cdot 10^{7} \mathrm{GeV}^{2}$, and $\mu=10^{-5} \mathrm{GeV}^{-1}$. Figures $8(\mathrm{~b})$ and 9 (b). The first loop cycle. $\varepsilon$ has relatively low value (Figure $9(\mathrm{~b})$ ) when the value of $\dot{x}^{1}$ is relatively high (Figure 8(b)). Figures 8(c) and 9(c). The second loop cycle, smaller than the first in the $\varepsilon, \dot{\varepsilon}$ graph and larger than the first in the $\dot{x}^{1}, \ddot{x}^{1}$ graph.

functions of the time iterations during the first loop cycle (Figures $8(\mathrm{~b})$ and $9(\mathrm{~b}))$. When the fluctuation occurs (around the iteration value of 2200), the Lyapunov exponent rises (relative instability). This is accompanied by a sudden rise in the velocity and the acceleration. However, the acceleration suddenly and very sharply jumps down and becomes negative. The powerful force which is responsible for the acceleration (brutal) change is the generalized Lorentz force. It is exactly at this point that the effective mass starts to grow (in absolute value), in order to reach a higher amplitude in its sinusoidal orbit. The effective mass is now growing, and at the same time the acceleration is decaying. The acceleration becomes less negative but slows the electron down, and the fluctuation terminates. Along with the slowing down, stability is restored (i.e., the Lyapunov exponent decays again).

To repeat, the effective mass markedly grows (in absolute value) immediately after the sharp change of the acceleration, which is caused by the generalized Lorentz force. Actually, the effective mass increases its fluctuation amplitude. As the mass grows the acceleration decays, and stability is restored. The growth of the effective mass should therefore be regarded as a "stabilizing reaction" of the electron against the generalized Lorentz force laid upon it. The effective mass generated in the vicinity of unity (the light cone) is very large (The effective mass value at $\varepsilon \approx 0.999$ is about 300 times greater than its value at $\varepsilon \approx 0.84$.) 


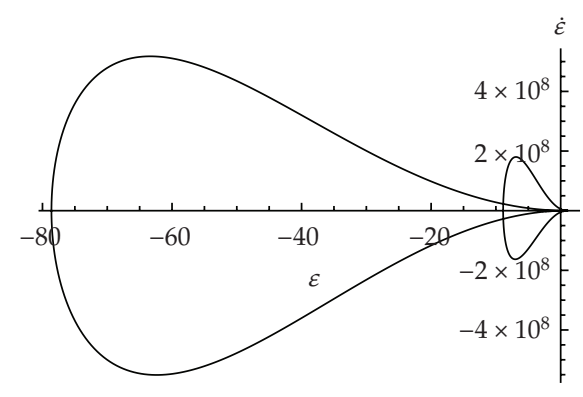

(a)

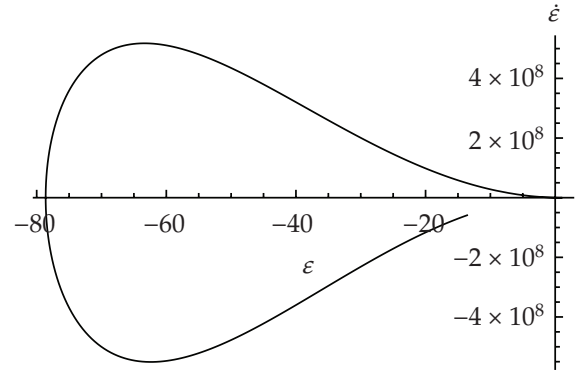

(b)

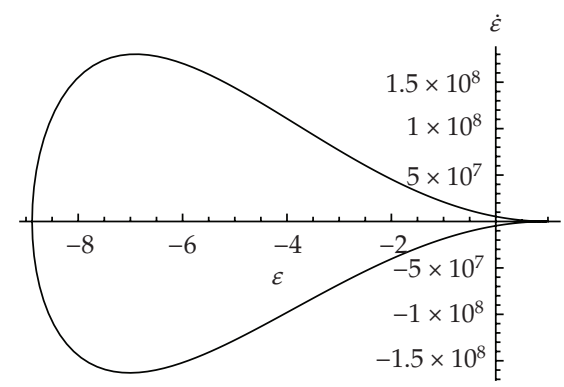

(c)

Figure 9: See the caption of Figure 8 which applies to both Figures 8 and 9.

As a second example, of which the motivation for presentation was discussed in the former section, we present a smooth, nonfluctuant orbit. This orbit has the same initial conditions and parameters as the former, fluctuant one. However, its cutoff frequency is higher: $10^{-1} \mathrm{GeV}^{-1}$. The higher cutoff frequency removes the fluctuations and shows a clear connection between the effective mass growth and the decay of the velocity and the acceleration. Figures 11(a)-11(e) present $\varepsilon$, the acceleration, the velocity, Lyapunov exponent, and the effective mass of this orbit, respectively. The effective mass (absolute value) growth (Figure 11(e)) is accompanied by decaying acceleration and velocity (Figures 11(b) and 11(c), resp.) and growing stability (Figure 11(d)).

The growth of effective mass and stability on the one hand and the decay of acceleration and velocity on the other hand go together. This, again, should be regarded as a stabilizing effect, which occurs also near the light cone.

\section{An External Force Added}

When an external force of the form

$$
e_{0} f_{\text {ext5 }}^{\mu}=A \cos (\omega \tau)
$$

is added, the orbit equation (3.22) is

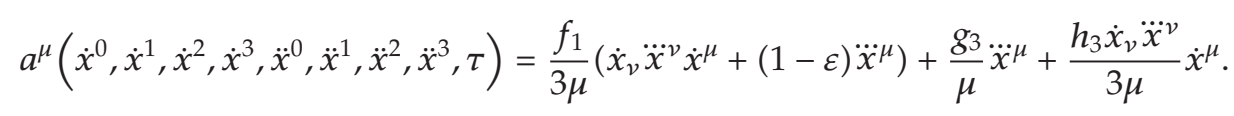




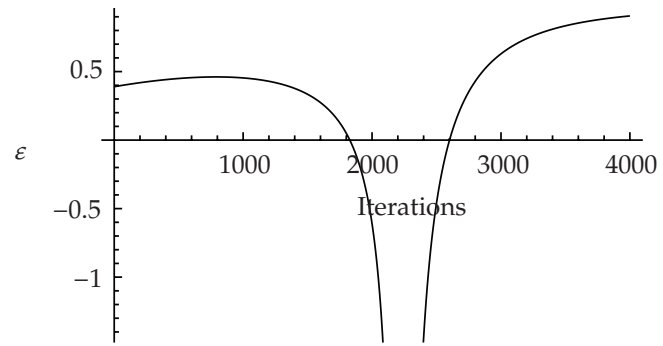

(a)

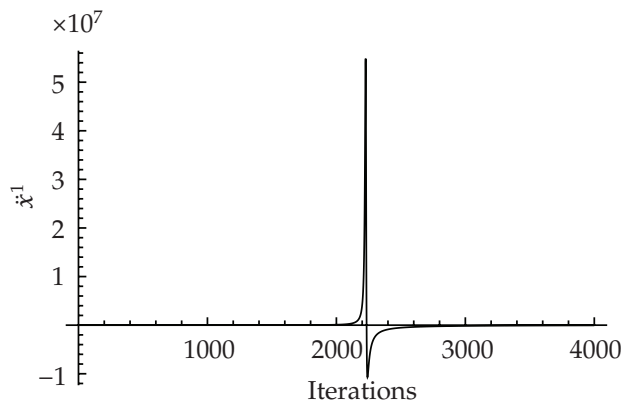

(c)

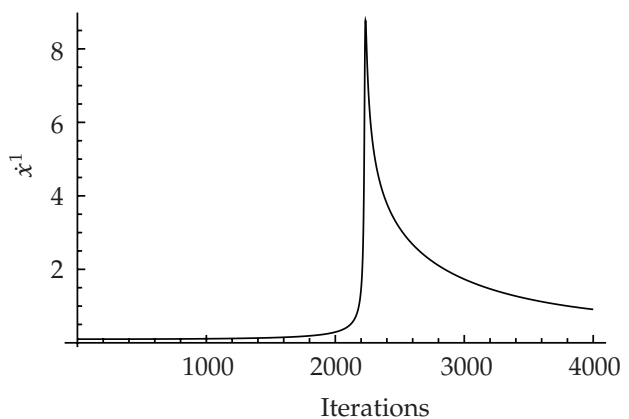

(b)

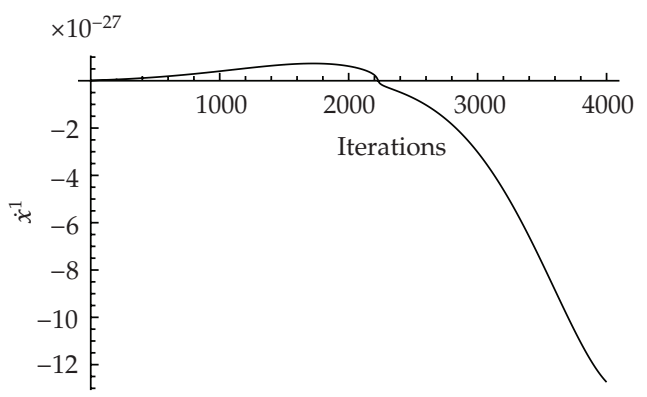

(d)

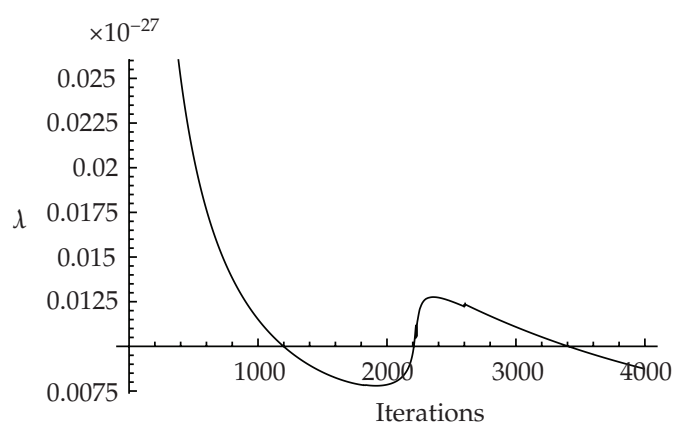

(e)

Figure 10: (a) $\varepsilon$ during the first loop cycle. (b) The $\dot{x}^{1}$ velocity during the first loop cycle. (c) The $\ddot{x}^{1}$ acceleration during the first loop cycle. (d) The effective mass $M(\varepsilon, \dot{\varepsilon})$ during the first loop cycle. (e) The Lyapunov exponent during the first loop cycle. 


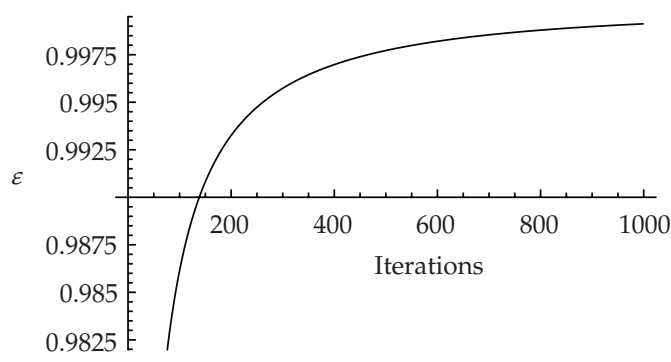

(a)

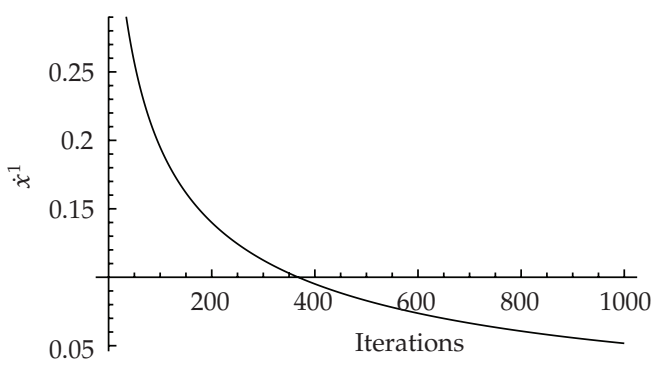

(c)

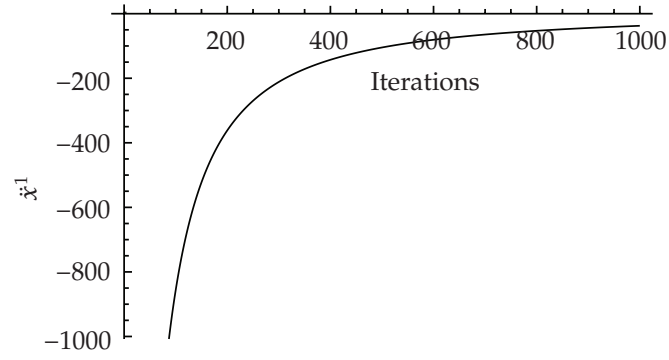

(b)

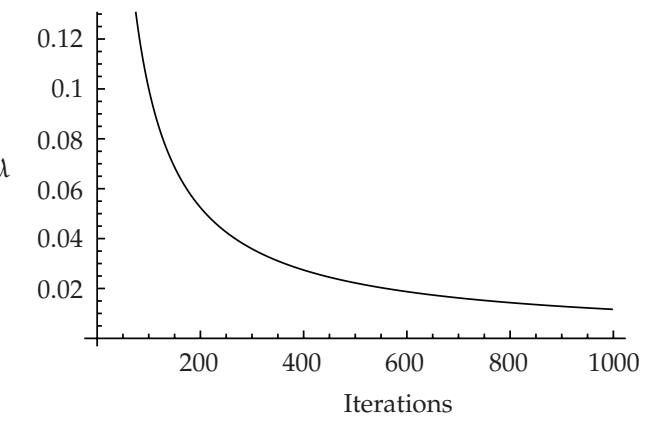

(d)

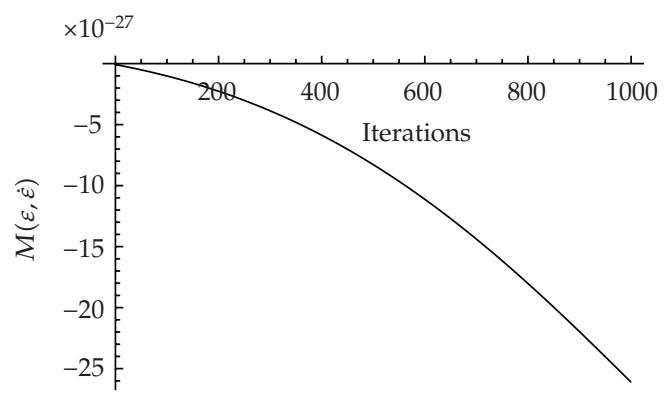

(e)

Figure 11: (a) and (b) $\varepsilon$ and the acceleration component $\ddot{x}^{1}$ as functions of the number of time iterations. (c) and (d) The velocity component $\dot{x}^{1}$ and the Lyapunov exponent as functions of the number of time iterations. (e) The effective (universal time) mass. 
This time we obtain the initial conditions $\dddot{x}^{\mu}$ as a function of the components of the first and second derivatives and also of $\tau$ explicitly. The expressions for $\dddot{x}^{\mu}$ have the same form as the $\tau$-independent solutions, with the only difference being in replacing $a^{\mu}$ from (5.2) with

$$
\begin{aligned}
a^{\mu}\left(\dot{x}^{0}, \dot{x}^{1}, \dot{x}^{2}, \dot{x}^{3}, \ddot{x}^{0}, \ddot{x}^{1}, \ddot{x}^{2}, \ddot{x}^{3}, \tau\right) \equiv & \frac{\left(M \cdot \ddot{x}^{\mu}-A \cos (\omega \tau)\right) \cdot \lambda(2 \pi)^{3}}{2 e_{0}^{2}} \\
& +\frac{1}{2}\left((1-\varepsilon) \ddot{x}^{\mu}+\frac{\dot{\varepsilon}}{2} \dot{x}^{\mu}\right)\left(\frac{f_{1}}{2 \mu^{2}}-\frac{\dot{\varepsilon} f_{1}^{\prime}}{2 \mu}\right)-\frac{g_{1}}{3 \mu^{3}} \dot{x}^{\mu} \\
& +\frac{g_{2}}{2 \mu^{2}} \ddot{x}^{\mu}+\frac{h_{1} \varepsilon}{2 \mu^{2}} \dot{x}^{\mu}-\frac{h_{2} \varepsilon}{\mu} \ddot{x}^{\mu}-\frac{h_{3} \ddot{x}_{v} \ddot{x}^{v}}{4 \mu} \dot{x}^{\mu}-\frac{h_{4} \dot{\varepsilon}^{2}}{8 \mu} \dot{x}^{\mu} .
\end{aligned}
$$

Again, the values for $\dot{x}^{\mu}$ and $\ddot{x}^{\mu}$ were chosen, and the values for $\dddot{x}^{\mu}$ were calculated using the orbit equation with the added perturbation (8.2). We used the initial values for $\dot{x}^{\mu}, \ddot{x}^{\mu}, \dddot{x}^{\mu}$ built in this way to program a Runge-Kutta algorithm as before. The program's output is a trajectory of a self-interaction attractor, as a function of the external perturbation. In Figure 12(a), we present an $(\varepsilon, \dot{\varepsilon})$ graph of the attractor's pure self-interaction, with the external perturbation turned off.

In Figure 12(b), the external perturbation, which we chose to be

$$
e_{0} f_{\mathrm{ext} 5}^{\mu}=1.6 \cdot 10^{-9} \cos \left(10^{14} \tau\right)
$$

was turned on. The time step in this figure is of order $10^{-9} \mathrm{GeV}^{-1}$. The figure shows a cycling polygonal orbit whose edges form an oval frame. The oval frame can be seen more clearly in Figure 12(c), where we removed the orbit lines which connect the coordinates.

As a large number of iterations are done, the polygonal orbit propagates in the path of the unperturbed attractor (Figure 12(d)).

When we use a different time step which is ten times smaller than the one in Figures 12(b)-12(d), we see again a cycling orbit whose edges form an oval (Figure 13(a)), but this time the oval frame is "filled" with a different polygonal pattern and looks like a "stretched" version of the previous one. As for the scale in Figure 13(a), it is about 12 times smaller than the one in Figures 12(b)-12(d). As before, there is a sinusoidal orbit when more iterations are taken (Figure 13(b)) and the propagation is in the path of the pure self-interaction attractor of Figure 12(a).

Two more similar reductions of the time step result in smaller-scale oval patterns, which are similar to the larger ones (Figures 14(a), 14(b) and 15(a), 15(b)). Here, again, the sinusoidal nature appears. This self-similarity on smaller time steps (and smaller scales) is a fractal feature and hence may suggest the existence of a strange attractor.

\section{Summary and Discussion}

We have shown that a generalized Lorentz force can be derived directly from the Hamiltonian for the classical relativistic motion of a charged particle in interaction with a generalized electromagnetic field by means of the Hamilton equations. 


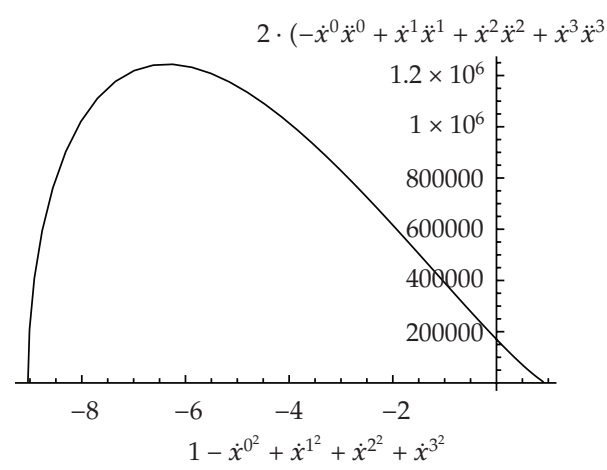

(a)

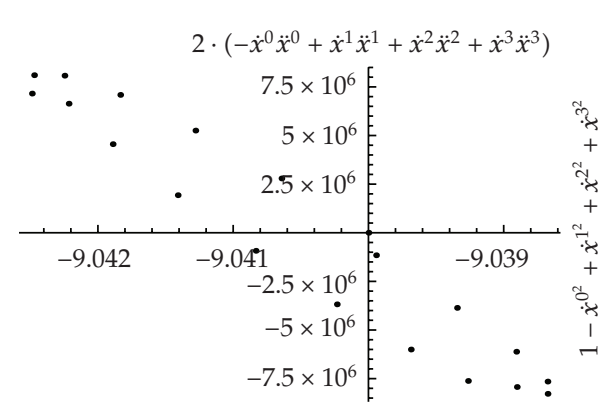

(c)

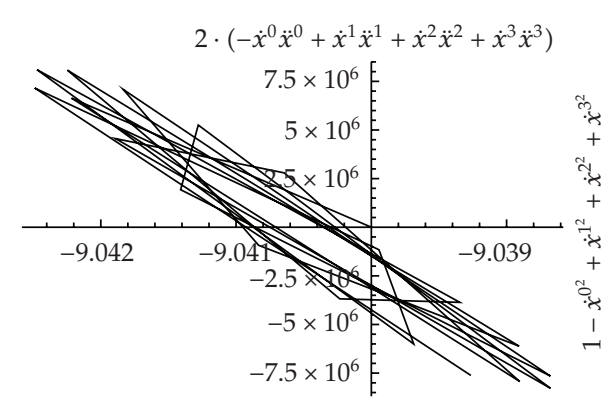

(b)

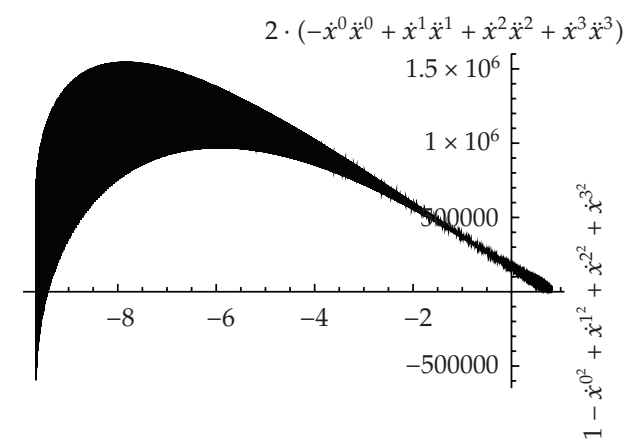

(d)

Figure 12: (a) The orbit without an external field. Initial conditions: $\dot{x}^{0}=3.2, \dot{x}^{1}=0.4, \dot{x}^{2}=0.2, \dot{x}^{3}=0.00049$, $\ddot{x}^{0}=2.83, \ddot{x}^{1}=0.3, \ddot{x}^{2}=0.1, \ddot{x}^{3}=-0.0011, M / \alpha=10^{5} \mathrm{GeV}^{2}$, and $\mu=10^{-5} \mathrm{GeV}^{-1}$. (b) and (c) The external perturbation added. The edges of the cycling polygonal orbit (b) form an oval frame, which can be seen as the lines connecting the coordinates are removed (c). Time step: $10^{-9} \mathrm{GeV}^{-1}$. (d) The propagation of the perturbate attractor after many time iterations is in the path of the pure self-interaction attractor of (a).

We computed the charged particle self-interaction and obtained differential equations for its motion by using the Green's function for the electromagnetic fields, and by taking the motion of the particle as the current source. We studied the motion both in the absence and in the presence of an external field. We introduced the quantity

$$
\varepsilon=1+\dot{x}^{\mu} \dot{x}_{\mu}
$$

which measures the deviation of the charged particle from its "mass shell".

\subsection{In the Absence of an External Field}

In the absence of an external field, we obtained an autonomous equation for the development of $\varepsilon$. This equation describes the mass deviation which is caused by pure self-interaction. Alongside with the autonomous equation, we also derived the four-component equation of motion (which we termed the orbit equation). Their compatibility was demonstrated by solving them numerically using consistent initial values. 


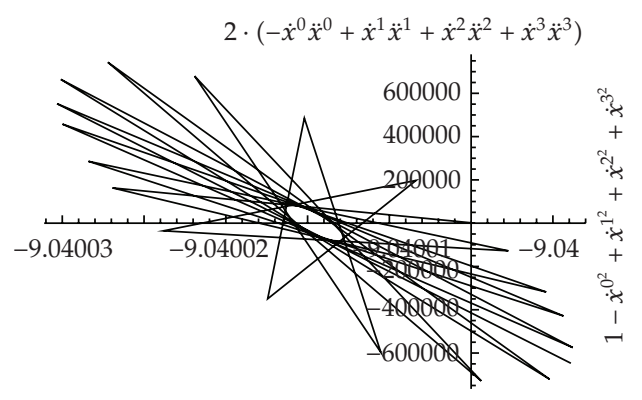

(a)

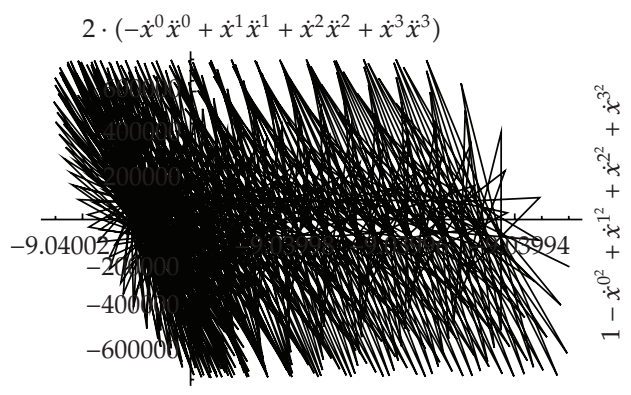

(b)

Figure 13: The smaller scale polygonal orbit and its propagation. One can observe the cycling polygonal orbit "filling" the oval frame in a star-like pattern (a). There is also a sinusoidal propagation (b). Time step: $10^{-10} \mathrm{GeV}^{-1}$. The scale is about 12 times smaller than the one in Figures 12(b)-12(d).

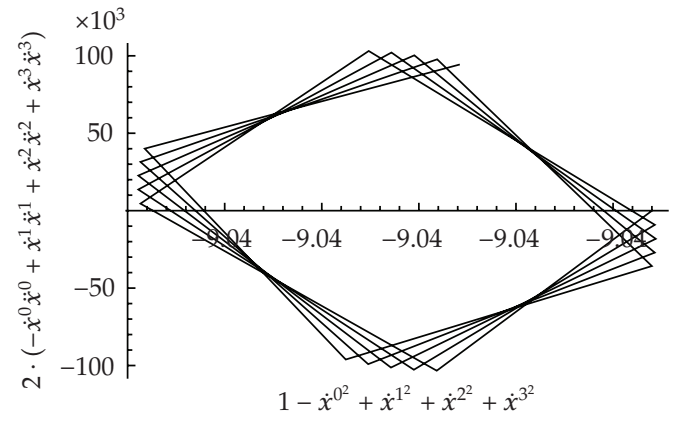

(a)

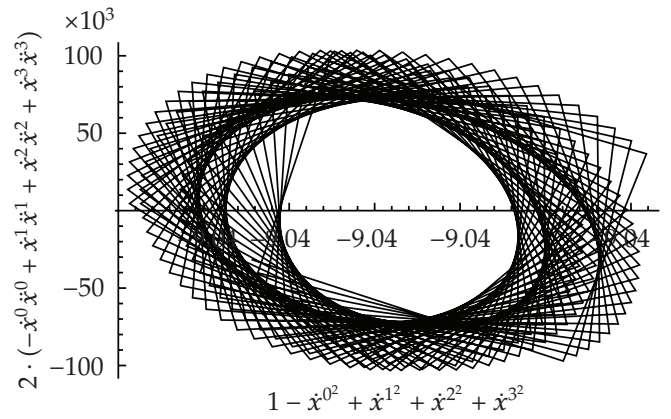

(b)

Figure 14: The cycling orbit filling the oval frame and the beginning of its propagation. Time step: $10^{-11} \mathrm{GeV}^{-1}$. The scale is about 6 times smaller than the one in Figures 13(a) and 13(b).

Both the autonomous equation and the orbit equation show the existence of an attractor. We investigated the accumulation area of the attractor-the vicinity to the phase space coordinate $(\varepsilon, \dot{\varepsilon}, \ddot{\varepsilon})=(1,0,0)$. We demonstrated that a lower and more "physical" cutoff frequency results in fluctuations in the orbit, as opposed to a higher (less physical) cutoff frequency, which results in a smooth orbit. Each fluctuation (or cycle, as it is seen in a phase space graph), causes a temporary instability, and a fluctuating attractor has shorter life span than a nonfluctuating one.

A lower cutoff frequency leads to $M(\varepsilon, \dot{\varepsilon})$ fluctuating with increasing amplitude as the orbit propagates towards the light cone. Within each such fluctuation the Lyapunov exponent jumps when the effective mass is relatively close to zero and decays when the absolute value of the effective mass increases. This phenomenon was demonstrated in three consecutive cycles.

A higher cutoff frequency removes the cycles/fluctuations and causes the absolute value of the effective mass to grow with increasing rate as $\varepsilon$ propagates towards the light cone. In this scenario, the Lyapunov exponent decays steadily and without jumps (Figure 11(d)) and may become negative (Figure $7(\mathrm{c})$ ).

In both cases mentioned above, a very large effective mass is generated as $\varepsilon \approx 1$ (Figures 3(f), 7(e), and 11(e)). This feature is possibly associated with effective nonzero size. 


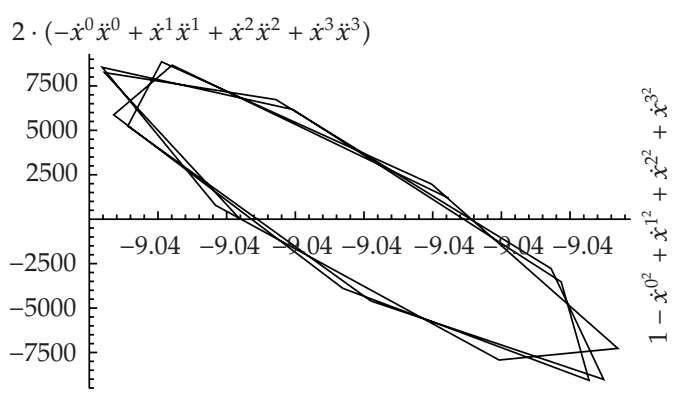

(a)

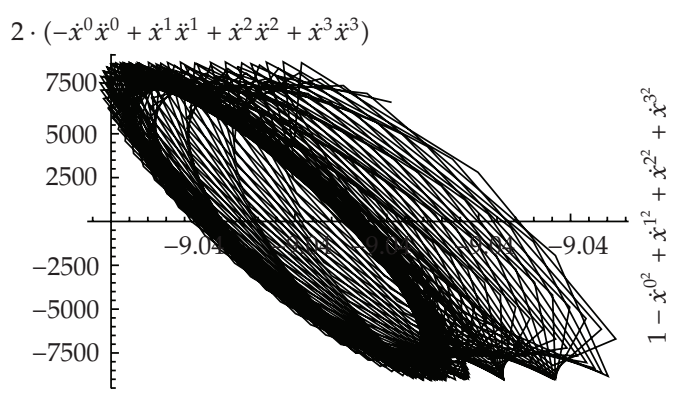

(b)

Figure 15: Time step: $10^{-12} \mathrm{GeV}^{-1}$. The scale is about 13 times smaller than the one in Figures $14(\mathrm{a})$ and 14(b).

The numerical solution of the orbit equations shows that the velocities and accelerations attained by the electron become more extreme with each fluctuation as $\varepsilon$ propagates towards unity. The acceleration, which is shown in Figure 10(c), increases at the beginning of a fluctuation and then suddenly and forcefully changes direction and reduces the velocity of the electron (Figure 10(b)). This powerful and sudden change is caused by the generalized Lorentz force. The slowing down of the electron is accompanied by a markedly growing effective mass, which stabilizes the electron against that force (Figures 10(d) and $10(e))$. The velocity decay along with the growing effective mass and stability is also seen in the nonfluctuating case (Figures 11(a)-11(e)).

We also checked whether the attractor has chaotic nature, using a Lyapunov exponent calculation and a time series analysis. The results did not indicate chaotic nature.

\subsection{In the Presence of External Field}

In the presence of an external field, the attractor acquires a remarkable stochasticity which may suggest fractal nature. The propagation nature of the attractor changes from a flat line propagation in the pure self-interaction case to a spring like, sinusoidal propagation of a cycling polygon in the external perturbation case (Figures 12, 13, and 14). However, the general path of the propagation remains the path of the pure self-interaction propagation (Figure 12(d)). A polygonal pattern also appears as smaller time steps are taken and the scales are smaller. This feature of self-similarity in smaller scales is of fractal nature and may suggest the existence of a strange attractor. Its stochastic type signals may be found in experiments with high-frequency detectors.

\section{References}

[1] M. Abraham, Theorie der Elektrizitat. II. Elektromagnetische Theorie der Strahlung, B.G. Teubner, Leipzig, Germany, 1905.

[2] P. A. M. Dirac, "Classical theory of radiating electrons," Proceedings of the Royal Society of London A, vol. 167, pp. 148-169, 1938.

[3] A. A. Sokolov and I. M. Ternov, Radiation from Relativistic Electrons, American Institute of Physics Translation Series, American Institute of Physics, New York, NY, USA, 1986.

[4] J. M. Aguirregabiria, "Solving forward Lorentz-Dirac-like equations," Journal of Physics A, vol. 30, no. 7, pp. 2391-2402, 1997. 
[5] F. Rohrlich, Classical Charged Particles, Addison-Wesley, Redwood City, Calif, USA, 1965.

[6] D. Villarroel, "Solutions without preacceleration to the one-dimensional Lorentz-Dirac equation," Physical Review A, vol. 55, no. 5, pp. 3333-3340, 1997.

[7] F. Rohrlich, "The unreasonable effectiveness of physical intuition: success while ignoring objections," Foundations of Physics, vol. 26, no. 12, pp. 1617-1626, 1996.

[8] F. Rohrlich, “Classical self-force," Physical Review D, vol. 60, no. 8, Article ID 084017, 5 pages, 1999.

[9] F. Rohrlich, "The self-force and radiation reaction," American Journal of Physics, vol. 68, no. 12, pp. 1109-1112, 2000.

[10] E. C. G. Stueckelberg, "La méchanique du point matériel en théorie de relativité et en théorie des quanta," Helvetica Physica Acta, vol. 15, pp. 23-37, 1942 (French) (French).

[11] E. C. G. Stueckelberg, "Remarque à propos de la création de paires de particules en théorie de relativité," Helvetica Physica Acta, vol. 14, pp. 588-594, 1941 (French).

[12] R. P. Feynman, "Mathematical formulation of the quantum theory of electromagnetic interaction," Physical Review, vol. 80, no. 3, pp. 440-457, 1950.

[13] J. Schwinger, "On gauge invariance and vacuum polarization," Physical Review, vol. 82, no. 5, pp. 664-679, 1951.

[14] L. P. Horwitz and C. Piron, "Relativistic dynamics," Helvetica Physica Acta, vol. 46, no. , pp. 316-326, 1973.

[15] L. P. Horwitz, "The unstable system in relativistic quantum mechanics," Foundations of Physics, vol. 25 , no. 1 , pp. 39-65, 1995.

[16] M. C. Land, N. Shnerb, and L. P. Horwitz, "On Feynman's approach to the foundations of gauge theory," Journal of Mathematical Physics, vol. 36, no. 7, pp. 3263-3288, 1995.

[17] D. Saad, L. P. Horwitz, and R. I. Arshansky, "Off-shell electromagnetism in manifestly covariant relativistic quantum mechanics," Foundations of Physics, vol. 19, no. 10, pp. 1125-1149, 1989.

[18] N. Shnerb and L. P. Horwitz, "Canonical quantization of four- and five-dimensional U(1) gauge theories," Physical Review A, vol. 48, no. 6, pp. 4068-4074, 1993.

[19] O. Oron and L. P. Horwitz, "Classical radiation reaction off-shell corrections to the covariant Lorentz force," Physics Letters A, vol. 280, no. 5-6, pp. 265-270, 2001.

[20] J. D. Jackson, Classical Electrodynamics, John Wiley \& Sons, New York, NY, USA, 2nd edition, 1975.

[21] O. Oron and L. P. Horwitz, "Eikonal approximation to 5D wave equations and the 4D space-time metric," Foundations of Physics, vol. 33, no. 9, pp. 1323-1338, 2003. 


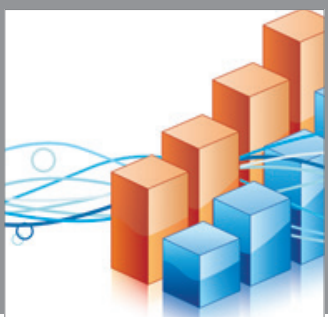

Advances in

Operations Research

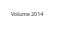

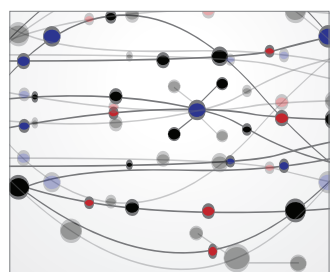

\section{The Scientific} World Journal
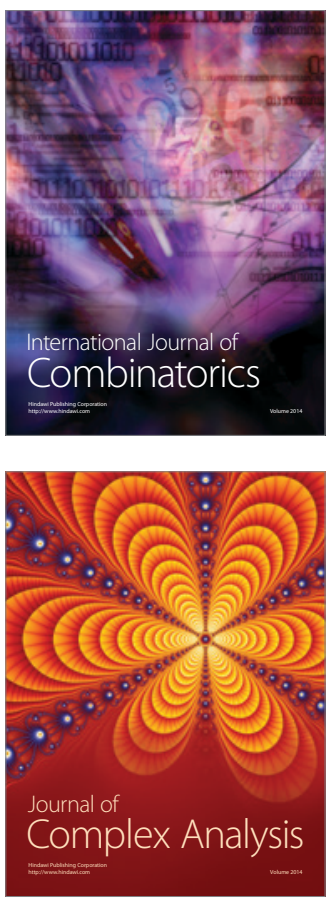

International Journal of

Mathematics and

Mathematical

Sciences
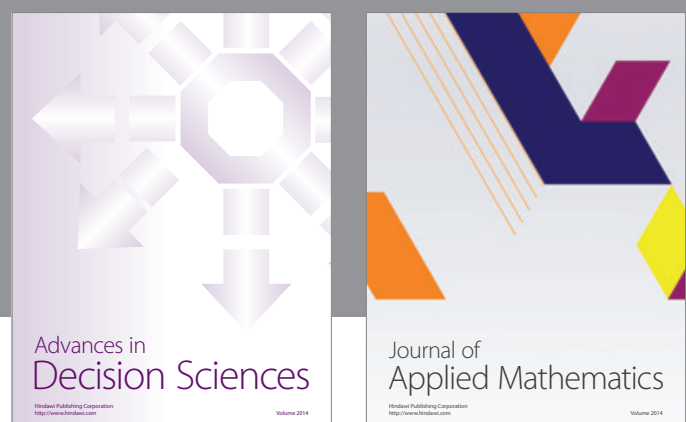

Journal of

Applied Mathematics
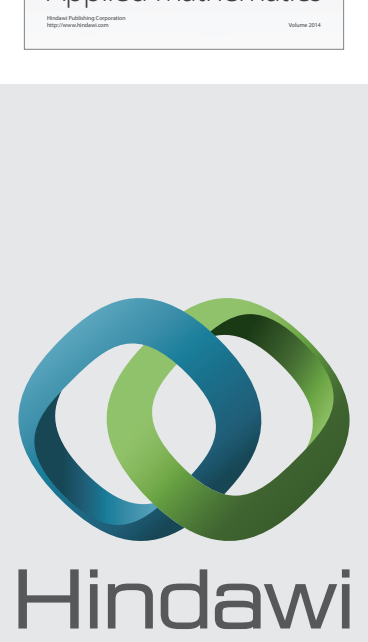

Submit your manuscripts at http://www.hindawi.com
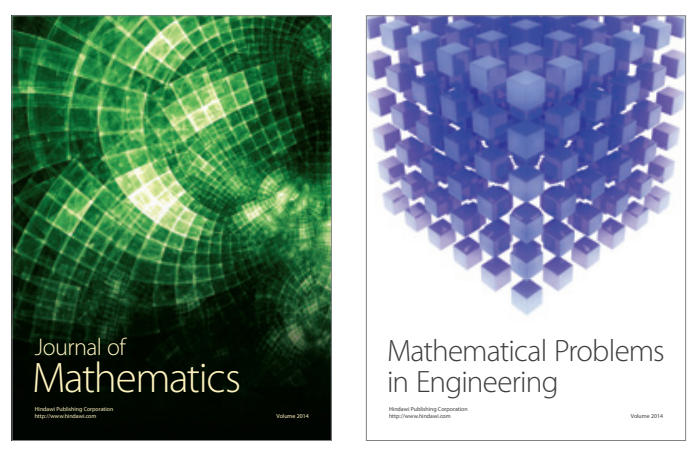

Mathematical Problems in Engineering
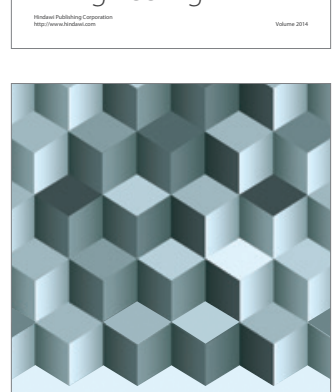

Journal of

Function Spaces
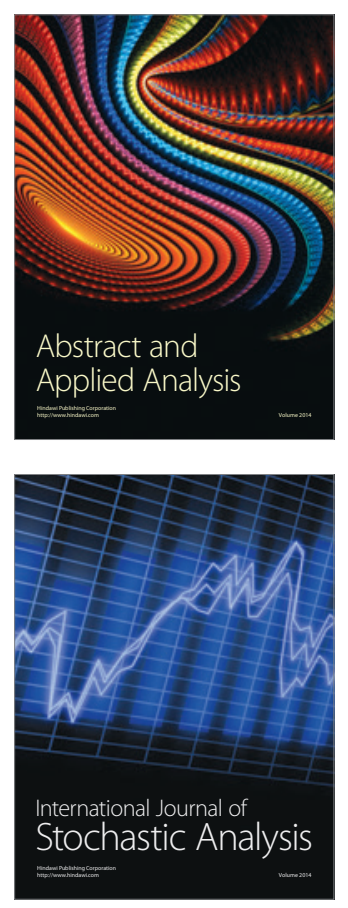

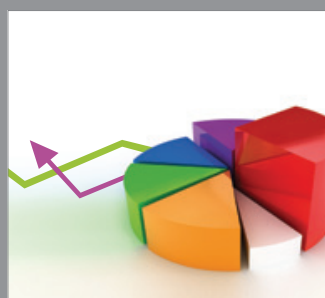

ournal of

Probability and Statistics

Promensencen
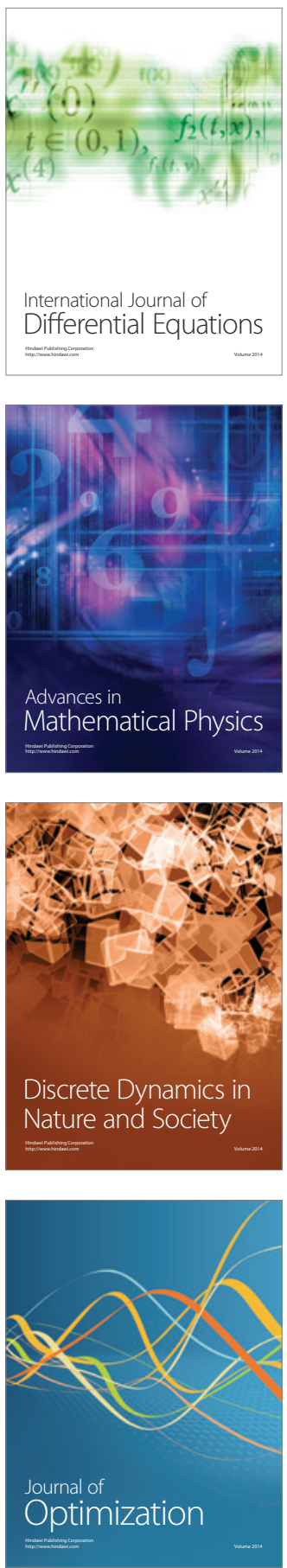